PNL-8518

UC-510

\title{
SIMILARITY ANALYSIS APPLIED TO THE DESIGN OF SCALED TESTS OF HYDRAULIC MITIGATION METHODS FOR TANK 241-SY-101
}

L. M. Liljegren

February 1993

Prepared for the U.S. Department of Energy under Contract DE-ACO5-76RLO 1830

Pacific Northwest Laboratory

Richland, Washington 99352 


\section{SUMMARY}

The purpose of this study conducted by Pacific Northwest Laboratory (PNL) is to obtain dimensionless parameters that can be used to design scaled tests of hydraulic mitigation strategies for Tank 241-SY-101 (SY-101) and to promote preliminary recommendations for the design of scaled tests of hydraulic mitigation strategies. The final design of scaled experiments will require additional evaluations of the physical properties of waste in SY-101.

The parameters governing the behavior of the tank contents during hydraulic mitigation are provided in Table S.1. Scaled tests are recommended in which the magnitudes of these parameters match the magnitudes that will be achieved during mitigation of SY-101 as closely as possible. The primary difficulty in implementing this recommended strategy is identified to be simultaneously matching Reynolds number and Froude number while testing in available $1 / 10^{\text {th }}$ or $1 / 12^{\text {th }}$ scale tanks. It is recommended that this first difficulty be overcome by performing tests using two simulants with differing viscosities. This will provide data that can be extrapolated to the correct Reynolds number, Froude number coordinate pair.

A second difficulty in implementing the recommended strategy is identifying the correct simulant properties required to match the dimensionless parameters that will be achieved in 101-SY during mitigation efforts. The sources of this difficulty are 1) the uncertainty in current measurements of properties in SY-101 and 2) the difficulties associated with manufacturing simulants that display specified properties.

Although the second difficulty cannot be overcome by application of scaling law analysis, two activities are recommended that would allow implementation of this analysis to the design of experiments: obtaining improved estimates of the physical properties in SY-101 and bench scale tests to develop simulants. The first is an ongoing tank safety activity at Hanford. The second should be included in the actual test plan for mitigation strategies for SY-101. 
TABLE S.1. Summary of Dimensionless Parameters and Recommendations for Scaled Testing

\begin{tabular}{|c|c|}
\hline $\begin{array}{l}\text { Froude number } \\
\text { Recommendation: Match Froude number by adjusting nozzle } \\
\text { velocity. }\end{array}$ & $\mathrm{Fr}^{2}=\frac{\left|\mathrm{W}_{0}\right|^{2}}{\mathrm{gH}_{0}}$ \\
\hline $\begin{array}{l}\text { Reynolds number based on tank height } \\
\text { Recommendation: If possible, adjust the effective kinematic } \\
\text { viscosity } \mu_{e o} \overline{p_{m}} \text { to match } R e \text { and } F r^{2} \text { simultaneously. (a) At } 1112^{\text {th }} \\
\text { scale it will be necessary to run at two values of kinematic viscosity } \\
\text { and extrapolate to target magnitude. }\end{array}$ & $\operatorname{ReH}_{\mathrm{H}}=\frac{\overline{\mathrm{Pm}_{\mathrm{m}}} \mathrm{W}_{\mathrm{o}} \mathrm{H}_{\mathrm{O}}}{\mu_{\mathrm{eo}}}$ \\
\hline $\begin{array}{l}\text { Inverse time parameter } \\
\text { Recommendation: Match parameter to SY-101 by adjusting period } \\
T_{0} \text { of oscillation. }\end{array}$ & $\eta_{t}=\frac{H_{o}}{T_{0} W_{0}}$ \\
\hline $\begin{array}{l}\text { Stress ratio } \\
\text { Recommendations: Match parameter by adjusting } \tau_{s s} / \overline{\rho_{m}} \text { of } \\
\text { simulant. }\end{array}$ & $N_{\tau}=\frac{\overline{P_{m}} W_{0}^{2}}{\tau_{s s}}$ \\
\hline $\begin{array}{l}\text { Settling velocity ratio } \\
\text { Recommendation: Match parameter by adjusting } V_{\text {soi }} \text { of simulant; } \\
\text { the major challenge is identifying the correct value in SY-101 }\end{array}$ & $\tilde{\mathrm{V}}_{\text {soi }}=\frac{\mathrm{V}_{\text {soi }}}{\mathrm{W}_{\mathrm{o}}}$ \\
\hline $\begin{array}{l}\text { Density ratio } \\
\text { Recommendation: Match ratio in } S Y-101 \text {. }\end{array}$ & $N_{p}=\Delta p / \overline{p_{m}}$ \\
\hline $\begin{array}{l}\text { Mean solids volume fraction } \\
\text { Recommendation: Match ratio in SY-101. }\end{array}$ & $\overline{\varepsilon_{s}}$ \\
\hline $\begin{array}{l}\text { Functional variation of settling velocity with concentration } \\
\text { Recommendation: Match ratio in SY-101. }\end{array}$ & $f_{s o}\left(\varepsilon_{s}\right)$ \\
\hline $\begin{array}{l}\text { Functional dependence of viscosity } \\
\text { Recommendation: Match ratio in SY-101. The major challenge is } \\
\text { identifying the correct functional form in SY-101. }\end{array}$ & $\mathrm{f}_{\mu}\left(\tilde{\Delta}, \overline{\varepsilon_{\mathrm{s}}}+\Delta \varepsilon_{\mathrm{s}}\right)$ \\
\hline
\end{tabular}

(a) Matching $\mathrm{Fr}^{2}$ and $\mathrm{Re}_{\mathrm{H}}$ simultaneously can be achieved by using a simulant with $\operatorname{Re}_{\mathrm{H}}^{2} / \mathrm{Fr}^{2}=\frac{{\overline{\rho_{\mathrm{m}}}}^{2} \mathrm{gH}_{\mathrm{O}}{ }^{3}}{\mu_{e_{\mathrm{o}}}{ }^{2}}$ equal to the value in the tank. 


\section{CONTENTS}

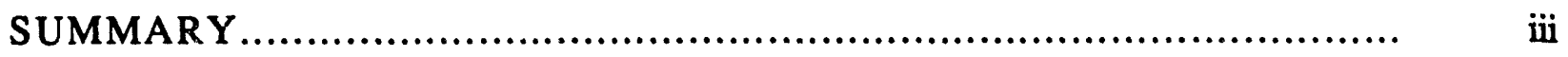

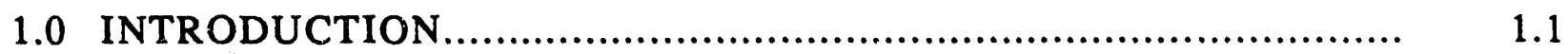

1.1 PURPOSE, SCOPE, AND METHOD ................................ 1.2

1.2 LIMITATIONS ...................................................... 1.2

2.0 GENERAL FEATURES OF HYDRAULIC MTTIGATION TECHNIQUES ...... 2.1

2.1 PROPOSED JET MIXING IMPLEMENTATIONS ....................... 2.1

2.1.1 Type of Pump Used to Achieve Mixing ............................. 2.2

2.1.2 Jet Orientation and Position ..................................... $\quad 2.3$

2.2 PHYSICAL MECHANISMS INVOLVED IN JET MIXING AND MOBILIZATION ........................................................... $\quad 2.4$

2.2.1 Mobilization of Settled Solids Using Jets......................... 2.4

2.2.2 Maintaining Solids Suspension U̇sing Jets ....................... 2.6

2.2.3 Dimensionless Parameters Governing Mixing ..................... $\quad 2.9$

3.0 SCALING ANALYSIS OF BEHAVIOR DURING JET MITIGATION

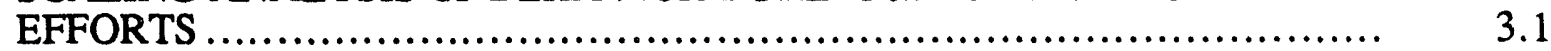

3.1 CONCEPT OF SIMILITUDE.............................................. 3.2

3.2 MATHEMATICAL MODELING ASSUMPTIONS ........................ 3.4

3.3 PRELIMINARY DEFINITIONS......................................... 3.5

3.3.1 Indicial Notation and Summation Convention........................ 3.5

3.3.2 Definition of Physical Parameters ................................... 3.5

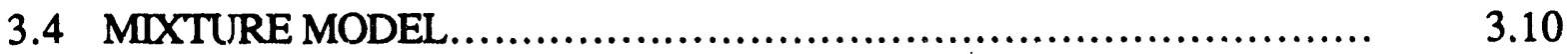

3.4.1 Continuity and Momentum Equations................................ 3.11

3.4.2 Boundary Conditions......................................... $\quad 3.12$

3.5 CHARACTERISTIC VELOCITIES, LENGTH SCALES, AND TIME

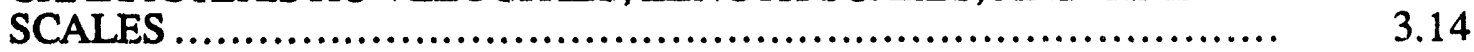

3.5.1 Length Scales............................................ $\quad 3.14$

3.5.2 Characteristic Velocities........................................ $\quad 3.15$ 
3.5.3 Characteristic Pressure .......................................... $\quad 3.16$

3.5.4 Characteristic Time Scale ........................................ $\quad 3.16$

3.6 GOVERNING DIFFERENTIAL EQUATIONS IN DIMENSIONLESS

FORM .................................................................. 3.16

3.6.1 Dimensionless Continuity and Momentum Equations................ $\quad 3.16$

3.6.2 Dimensionless Boundary Conditions ............................. $\quad 3.19$

3.6.3 Summary of Dimensionless Parameters .......................... $\quad 3.21$

4.0 EXPERIMENT DESIGN ................................................. 4.1

4.1 GENERAL DISCUSSION OF EXPERIMENTAL DESIGN ............... 4.1

4.2 PROPERTIES OF WASTE CONTAINED IN SY-101.......................... 4.3

4.2.1 Solids Volume Fraction ...........................................

4.2.2 The Density Ratio .............................................. 4.5

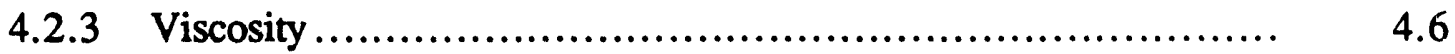

4.2.4 Particle Settling Velocity ......................................... 4.6

4.2.5 Shear Strength............................................. 4.7

4.3 ACHIEVING SIMILARITY IN SCALED EXPERIMENTS ............... 4.7

4.3.1 Properties Required at an Arbitrary Scale......................... $\quad 4.7$

4.3.2 Strategy for Small Scale Testing ................................. 4.13

5.0 REFERENCES ............................................................ 5.1

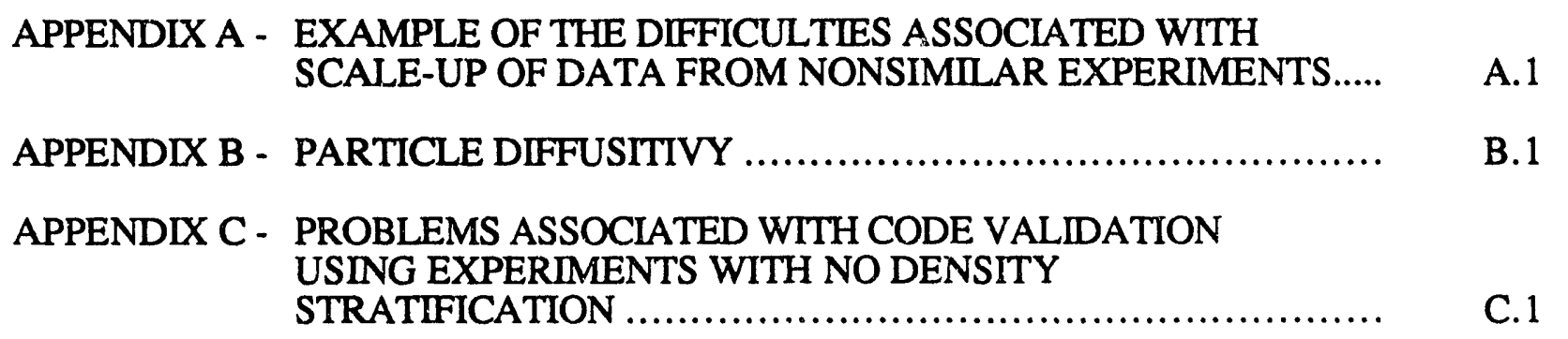




\section{TABLES}

S.1 Summary of Dimensionless Parameters and Recommendations for Scaled Testing

3.1 Summary of Dimensionless Parameters

4.1 Reported and Estimated Properties at $65^{\circ} \mathrm{C}$

4.4

4.2 Properties, Velocity, and Length Scale Factors Required to Achieve Similarity at $1 / 12^{\text {th }}$ and $1 / 4^{\text {th }}$ Scale 


\subsection{INTRODUCTION}

The episodic gas releases from Tank 241-SY-101 (SY-101) pose a potential safety hazard. It is thought that gas releases occur because gases are generated and trapped in layers of settled solids located at the bottom of the tank. Methods to temporarily mitigate the hazardous situation and to ultimately remediate the situation must be developed. Proposed mitigation technologies include mixing, heating, and ultrasound. The merits of these proposed mitigation technologies and the uncertainties associated with the potential success of these technologies are discussed in Babad et al. (1991).

This document focuses on issues associated with testing of hydraulic mitigation technologies proposed for SY-101. The basic assumption underlying the concept of hydraulic mitigation is that mobilization or maintained suspension of the solids settled in the bottom of the tank will prevent gas accumulation. Engineering of hydraulic technologies will require testing to determine the operating parameters required to mobilize the solids and to maintain these solids in suspension. Because full scale testing is extremely expensive (even when possible), scaled tests are needed to assess the merit of the proposed technologies and to provide data for numerical or analytical modeling.

Scaled tests will be conducted to determine the operating parameters required to mitigate conditions in SY-101 using hydraulic technologies, such as nozzle exit velocities. Pacific Northwest Laboratory (PNL) (a) performed this analysis to provide a basis for designing these tests. The results of this analysis will be used both to design the experiments and to scale the data obtained during tests. The scale-up procedure will allow the jet velocity required at full scaie to be predicted on the basis of scaled experimu.nts.

This research is conducted to support testing and evaluation of proposed hydraulic mitigation concepts only. The work here is oriented towards determining the jet velocities, nozzle sizes, and other operating parameters required to mobilize the settled solids in SY-101 and maintain them in suspension. Additional evidence to support or refute the basic premise that mobilization of the solids will prevent gas accumulation is being conducted in separate effor:s (Allemann et al. 1991; Allemann 1991).(b)

(a) Operated for the U.S. Department of Energy by the Battelle Memorial Institute under Contract DE-AC06-76RLO 1830.

(b) Allemann, R. T. 1991. Progress Report: Physical Modeling to Support Flammable Gas Waste Tank Mitigation. Unpublished Letter Report. Pacific Northwest Laboratory, Richland, Washington. 


\subsection{PURPOSE, SCOPE. AND METHOD}

The purpose of this effort is to support the design and interpretation of scaled tests of hydraulic mitigation technologies. Design of a specific jet mitigation technology requires information that allows prediction of

- jet velocity and flow rates required to initially suspend settled solids in SY-101

- jet velocity and flow rates required to prevent resettling at full scale.

After the velocity and jet flow rates required for each conceptual impiementation of jet mixing are determined, other design variables may be calculated. These include

- instantaneous power, flow rate, and velocity requirements

- continuous or intermittent operation of mixer pumps

- pump types (i.e., liquid piston, slurry mixer pumps).

The scope of this effort is to

- perform a similarity analysis that can be used to design experiments to determine the jet flow rates and velocities required to resuspend settled material and maintain it in suspension

- provide algebraic relationships which can be used to calculate the properties of a dynamic simulant to be used in a scaled experiment

- recommend a method of testing that will provide data that can be extrapolated to predict effectiveness of the equipment to be used in SY-101.

\subsection{LIMITATIONS}

Limitations are inherent to any analysis related to the mitigation of conditions in SY-101; the limitations for this analysis are categorized in four groups and are provided below.

1. Limitations in knowledge of the physical properties of tank contents

Analysis of samples taken from SY-101 provides limited information about its actual contents because:

- The tank contents are inhomogeneous. Properties vary throughout the tank, so individual core samples do not represent the average of the tank contents.

- The tank contents are disturbed during sampling. Properties such as shear strength may take time to develop. Consequently, the shear strength data may be inaccurate when used to determine the shear strength of sludge in SY-101. 
- It is difficult to measure some properties that are relevant to mixing. For example, ideally, the variation of effective viscosity with concentration and strain rate should be known over a large range of interest. It is not practical to perform measurements at numerous concentrations, and physical limitations of any rheometer limit measurement of viscosity to a finite strain rate range. Other properties that are difficult to characterize include density of the undissolved solids, volume fraction of the undissolved solids, and the unhindered settling velocity of the solids.

\section{Limitations to scaling law analysis}

Similarity law analysis is a mathematical technique that, in principl", has no limitations. In practice, any scaling law analysis is limited by the need for assumptions about physical mechanisms involved in the phenomena to be scaled. In this analysis, the scaling law analysis relies on a number of assumptions, which are provided in Section 3.2. Two important assumptions impose limitations on scaling law analysis:

- The resistance of the settled nonconvective layer to mobilization is assumed to be proportional to the shear strength.

- It is assumed that the slurry properties depend only on the mixture's solids concentration and the strain rate tensor; that is, it is assumed that properties such as viscosity or yield stress are not time dependent.

The assumption that properties in SY-101 are not time dependent may te unwarranted. It has been suggested that the speed of tank overturns indicates that the mixture may be thixotropic, which means that the effective viscosity or resistance to flow decreases with time. No analysis to confirm thixotropy or any other time dependent behavior has been performed on core samples. In addition, the speed of tank overtuins can be explained without requiring thixotropy. Scaling law results and, more importantly, de.iign parameters for mixing will differ for fluids that display thixotropy or any other time dependent behavior.

3. Limitations inherent to scope of this analysis

Scaling law analyses do not provide complete solutions of mixing behavior in a tank.

Rather, scaling law analysis provides the parameters required to design experiments properly and to describe how data may be scaled up from these experiments.

Scaling law analysis can only be used to scale up data from properly designed experiments. It will not be possible to use this analysis to predict the jet velocity required to mitigate SY101 on the basis of any scaled experiment that does not adhere to the principles of similarity outlined here. ${ }^{(a)}$

(a) An example of the difficulties associated with scale up of data from improperly designed experiments is provided in Appendix A. 


\section{Limitations related to the success of jet mixing}

The scope of this analysis is to determine operating parameters required to mobilize and resuspend solids using hydraulic methods, in particular jet mixing technologies. It is not anticipated that large scale testing with hazardous simulants which trap gas in a manner similar to waste in SY-101 will be possible. Consequently, it will not be possible to observe all features required to ensure that mixing will mitigate the conditions in SY-101. Hydraulic mitigation is expected to be successful if both of the following statements are true:

- Gas accumulation cannot occur when the nonconvective region is mobilized and maintained in suspension.

- The settled nonconvective layer is mobilized and maintained in suspension by hydraulic mitigation. 


\subsection{GENERAL FEATURES OF HYDRAULIC MITIGATION TECHNIOUES}

This section describes general features of proposed jet mitigation techniques for SY-101. These descriptions are limited to features that are relevant to similarity analysis; more detailed descriptions are in Babad et al. (1991). Section 2.1 discusses features of the proposed implementations of jet mixing technologies. Section 2.2 is a brief description of the mechanisms involved in jet mixing.

Currently, SY-101 contains a mixture of solid and liquid waste that appears to have stratified into two distinct regions. One region has a relatively low solids concentration and a low viscosity; it is a convective region in which convective cells form and permit convective heat transfer throughout the region. The other region has a relatively high solids concentration and is a nonconvective region in which the effective viscosity is sufficiently high to prevent the formation of these natural convection cells. The current consensus is that gas accumulates and is trapped in the lower nonconvective layer. This eventually results in an unstable density inversion that causes the entire settled layer, or some portion of the settled layer, to break away and rise to the upper regions of the tank. During this break away, or "overturn," process, large amounts of gas are suddenly released.

Mitigation using hydraulic jets has been proposed. It is thought that high velocity jets can disrupt the settled nonconvective layer sufficiently to prevent significant gas accumulation. The jets would first be activated just after a gas release event when the gas inventory is at a minimum. It is anticipated that less gas would be retained while jets are operated, and consequently, sudden gas releases will be prevented. However, the fraction of settled solids mobilized during a gas release, the total gas volume remaining in the tank after an event, and the amount of gas that can be retained by the mobilized solids are currently unknown.

\subsection{PROPOSED JET MIXING IMPLEMENTTATIONS}

The proposed jet mitigation technologies described by Babad et al. (1991) include vertical downward facing draft tubes and horizontal jets. Using engineering judgment to predict effectiveness, Babad et al. ranked proposed jet mixing mitigation methods in order of decreasing effectiveness: 1) horizontal jet, 2) vertical down-turned jet, 3) vertical upward jet, and 4) liquid piston. To verify the effectiveness and support these rankings, each method should be tested. Testing will also be required to obtain design information such as the flow rates required to maintain solids in suspension. 
Although the above hydraulic methods vary in specific geometry, all have many common features that are important for designing scaled experiments: during jet mixing, flow inside the tank involves the same mixing and resuspension mechanisms in all four methods. Therefore, identical fluid transport equations can describe the flow field in the tank during any hydraulic mitigation effort. The general requirements for designing experiments that provide data that can be scaled are the same for all proposed jet mitigation problems.

Features which differ between each proposed technology may be classified into two major categories. The first category is flow geometry; for example, jets may be horizontal or vertical, oscillating or stationary. The second category is the motive force, or method of achieving flow; motive force can be supplied by different pump designs, such as a centrifugal pump or a liquid piston.

Geometric features of the test cases must match those of the proposed technology to provide useful results. For instance, horizontal jets must be tested using horizontal jets. Different features may also affect the magnitude of the optimum operating parameters. For example, the optimum nozzle velocity for an opposed jet oscillating m: xer pump design may be different from the intermittent twelve nozzle liquid piston desis,

To provide a basis for understanding the range of proposed technologies, specific features of proposed technologies are briefly described below. The types of pumps proposed are described in Section 2.1.1. Section 2.1.2 describes the types of geometries proposed. More detailed descriptions are available in Babad et al. (1991).

\subsubsection{Type of Pump Used to Achieve Mixing}

Two methods of driving the jet he ve been proposed: mechanical pump or liquid piston. Either method may be used to drive horizontal jets or vertical jets, depending on the proposed technology.

The liquid piston uses pneumatic pressure to drive fluid from a reservoir out through orifices into the waste tank. Following expulsion, pressure is removed, fluid reenters the reservoir, and the cycle is repeated. Operation of the liquid piston is inherently time dependent.

Mechanical pumps are for techniques in which jets are operated continuously. One example of a pump driven design is an oscillating mixer pump. Two opposed jets emit from opposed nozzles, and the entire pump oscillates over $180^{\circ}$. Scaled testing requires similar pump oscillation, and the design of the scaled mixer pump should match the relevant features of the full scale pump.

The method of driving the jet affects scaled testing indirectly. For instance, periodicity of the jets must be replicated during testing. Liquid piston designs should be tested using periodic 
suction and expulsion; oscillating mixer pumps should be tested with an appropriate osciliation pattern. In both test configurations, the nozzle and pump design must replicate the flow conditions at the nozzle exit.

\subsubsection{Jet Orientation and Position}

A number of jet directions and locations have been proposed. The geometries may be classified into horizontal jets, upwand vertical jets, and downward vertical jets. The geometries that have been proposed are described below.

\subsubsection{Horizontal Jets}

In this geometry, solids are mobilized and maintained in suspension by horizontal jets that scour the tank floor. Examples of some technologies which use horizontal jet technique are

- oscillating mixer pumps-In this configuration, the jets emit from two opposing nozzles; slurry intake to feed the jets is above the exit nozzles, and a centrifugal pump is required to drive the jet. The entire tank floor is swept during one oscillation of the jet.

- radially driven jets-In this configuration, twelve radially driven jets emit horizontally. Continuous operation of the jets would require a separate fluid intake; intermittently operated jets could be driven using a liquid piston which takes in and emits fluid from a single intake.

\subsubsection{Vertical Jets}

Vertical geometries have also been suggested for use in mitigation strategies. An example is a downward facing draft tube. In this application, fluid enters the draft tube near the top of the tube and leaves the bottom as a downward facing jet. In an alternative version, the draft tube faces upward.

A number of parameters are unspecified in nearly all conceptual designs of mitigation strategies. Some parameters that may affect the success of a mitigation strategy include

- the distance of the jets from the tank floor

- the location of the fluid intake relative to the location of the jet

- nozzle geometry.

Because most of the proposed mitigation strategies are in the conceptual state, few of these parameters are described in documents such as Babad et al. (1991). Geometric similarity, which will be 
discussed in Section 3.1, 1eiluires that test fixtures be geometrically scaled versions of those which will be used in SY-101. Cunsequently, the geometric features of proposed hydraulic methods will require final definition before testing.

\subsection{PHYSICAL,MECHANISMS INVOLVED NN JET MIXING AND MOBILIZATION}

An understanding of mobilization and mixing is important to this analysis because each describes a stage required during mitigation of SY-101 using jet mixing technologies. This section discusses physical phenomena associated with mobilization and mixing.

The first stage is mobilization of the sludge layer at the tank bottom; the second aintaining these solids in suspension until a time when the slurry can be removed from the tank. It is also possible that mobilization will be accomplished by a naturally induced overturn; if so, a method of maintaining solids in suspension will still be required. In any case, separate physical mechanisms are involved in the solids mobilization and suspension.

Dimensionless parameters describing individual physiral phenomena involved in each step of mitigation are identified. They are similar to those obtained in the mathematical analysis in Section 3.7. Unfortunately, all dimensionless parameters cannot be efficiently identified by simply describing a number of individual mechanisms. This is because 1) there is overlap in the parameters identified because some parameters obtained by examining individual mechanisms can be shown to be algebraically redundant in the sense that they are linear combinations of others parameters and 2) it is possible to omit an important mechanism and overlook an individual parameter.

A more efficient method of identifying all relevant dimensionless scaling parameters, while ensuring that redundant parameters are not included, is to identify the governing equations that describe flow throughout the tank and then mathematically manipulate these equations to obtain the relevant dimensionless parameters. However, even technically trained individuals have difficulty understanding the physical mechanisms involved during flow applications on the basis of the mathematical method aioicic. Consequently, it is useful to include both physical and mathematical arguments despite the fact that to do so is redundant.

\subsubsection{Mobilization of Settled Solids Using Jets}

Two mechanisms for sludge mobilization have been described by Powell et al. (1990); jets mobilize solids by

- erosion by shearing action of high velocity fluid moving parallel to the surface of a sludge bank 
- bulk mobilization of sludge due to the impact of a high velocity jet normal to the sludge bank. This results in undercutting or displacement of ine bank.

It is not known a priori which mechanism will dominate during mitigation efforts. The mechanism whith will be favored in a particular physical situation will depend on a number of factors, inclung the ve'. $x$ city of the fluid near the sludge bank, the shape of the sludge bank, the size of particles in the sludge bank, solids and gas concentrations, and the inter-particle forces between solids in the sludge bank. Many of these factors are dependent parameters during tests; for example, the jet action will form the sludge bank and influence its shape. It is also possible that erosion will be important during some stages of mobilization, while bulk mobilization will be important in others.

Powell et al. (a) suggested that the shear strength of the sludge describes the ability of the sludge to resist mobilization. Based on the assumption the the shear strength described the resistance of the sludge to mobilization, they proposed two specific functional forms for the variation of the diameter of the maximum circle that can be cleared by an oscillating Newtonian jet. One functional form describes mobilization by erosion and one describes bulk mobilization. Both analyses were based on the idealized case in which a two dimensional boundary layer flowed past an infinite sludge bank. Other factors wilich could affect the functional form during actual mitigation efforts include the spread rate of the jet and the degree of buoyancy of the jet, which would affect the location at which the jet first impacts the sludge bank.

Both correlations proposed by Powvell et al.(a) agree with a more general statement, which is that the ratio of maximum circle diameter to the nozzle diameter is a function of the jet Reynolds number and a yield parameter:

$$
\frac{\mathrm{ECR}}{\mathrm{D}_{\mathrm{o}}}=f\left(\mathrm{Re}_{\mathrm{D}}, \mathrm{N}_{\tau}\right)
$$

$$
\begin{aligned}
\text { where } N_{\tau}=\frac{\rho_{m} W_{0}^{2}}{\tau_{s S}} & =\text { a yield parameter } \\
\operatorname{Re}_{\mathrm{D}}=\frac{\rho_{m} W_{0} D_{0}}{\mu} & =\text { the jet Reynolds number } \\
E C R & =\text { the "effective cleaning radius" or the maximum diameter } \\
\text { of a circle cleared by the jet } & \\
W_{0} & =\text { the jet nozzle veiixcity }
\end{aligned}
$$

(a) Powell, M. R., C. L. Fow, G. A. Whyatt, P. A. Scott, and C. M. Ruecker. 1990. Proposed Test Strategy for the Evaluation of Double-Shell Tank Sludge Mobilization. Draft report. Pacific Northwest Laboratory, Richland, Washington. Cited hereafter as Powell et al. (1990 draft). 


$$
\begin{aligned}
t_{s s} & =\text { the shear strength of the sludge } \\
\mu & =\text { the absolute viscosity of the fluid } \\
D_{0} & =\text { the diameter of the jet nozzle } \\
\rho_{m} & =\text { the fluid density } \\
f & =\text { represents some arbitrary function. }
\end{aligned}
$$

Fxperiments in which the yield parameter was tested in the range anticipated during operation of mixer pumps in double shell tanks were conducted by Powell et al. (1990 draft). Data collected during these experiments were analyzed by Powell et al. to determine the cleaning radius using real mixer pumps. Results indicated that during mobilization of clay/water simulants and silica soda ash simulants, the "ECR" (i.e., the radius cleaned by a jet nozzle) was a function of the yield parameter. However, the cleaning radius of polymer simulants such as kaolin/Ludox could not be predicted using shear strength.

Investigations of sludge mobilization are continuing. Although Powell(a) has recently suggested that tensile strength may be a more useful parameter for characterizing sludge resistance, this suggestion has not yet been tested.

\subsubsection{Maintaining Solids Suspension Using Jets}

Two basic mechanisms are important during solids suspension: 1) large-scale circulation flow patterns and 2) small-scale diffusive motion. General descriptions of the two mechanisms and scaling issues associated with each are provided here.

Mixing is expected to occur in SY-101 as a result of large-scale circulation patterns induced by jets; these patterns include the large scale upward motion of a particle laden jet in some region of the tank. Particles may be transported to the upper regions of a tank by a coherent jet if 1 ) the jet is particle laden and 2) the jet is capable of traveling to the upper portions of the tank.

In most proposed implementations of jet mixing, a jet is located near the lower regions of the tank. The jet pump or jet piston takes in particle laden fluid and emits this fluid at high velocity. The jet is then expected to travel to the upper regions of the tank. Eventually, the jet loses momentum, and particles are expected to fall by gravity to lower regions of tank.

This scenario will actually occur if the particle laden jet is capable of traveling to the upper regions of the tank. However, a large scale circulation pattern could be seriously affected by slight density differences in the tank. Because the jet is particle laden, its average density will be a func-

(a) Personal communication from M. R. Powell to L. M. Liljegren on April 17, 1992. 
tion of the particle loading. If the jet intake is from the bottom of the tank, the density of the jet will be greater than the density of the surrounding fluid. The excess density relative to the surrounding fluid will increase as the jet rises to the upper regions where particle loading is lower.

Excess density combined with low momentum can have a dominant influence on the jet motion. When a dense jet emits upward, gravity tends to retard the jet. The jet will only reach the upper regions if its momentum is sufficiently large enough to overcome the force of gravity. The height to which a jet will rise may be stated in general functional form as

$$
\frac{\mathrm{H}_{\mathrm{j}}}{\mathrm{D}_{\mathrm{o}}}=f\left(\mathrm{Fr}, \mathrm{N}_{\rho \mathrm{j}}, \Theta, \mathrm{Re}_{\mathrm{Do}}\right)
$$

where $\mathrm{H}_{\mathrm{j}}{ }^{\cdot}=$ the maximum vertical distance traveled by the jet

$\Theta=$ the jet angle with respect to vertical

$\mathrm{Fr}^{2}=\frac{\mathrm{W}_{\mathrm{O}}^{2}}{\mathrm{gD}_{\mathrm{O}}}=$ the jet Froude number based on the jet diameter

$\mathrm{N}_{\rho j}=\frac{\Delta \rho}{\rho j}=$ the relative density difference between the jet and the surrounding fluid

$\rho j=$ the jet density ai

$\Delta \rho=$ the density di."erence between the jet and the surrounding fluid.

When the relative density difference of the jet is large and Froude number is low, the jet motion will be strongly influenced by gravity. In the case of jet mixing, both the density of the jet at the nozzle and the density difference between the jet and the surrounding fluid will depend on the degree of mixing achieved.

The example above is used to describe the potential influence of gravity on large scale motions and to discuss the parameters which can affect the large scale motion. The specific features of the flow will differ for buoyant jets; but the correct scaling parameters $\left(\mathrm{Fr}, \mathrm{N}_{\rho}, \operatorname{Re}_{\mathrm{Do}}\right.$, etc.) would be identical.

Different scaling laws govern the mixing by small scale diffusive motions. Diffusion of particles occurs as a result of the random actions of small scale eddies that induce a net flux of particles from regions of high concentration to regions of low concentrations. This is conceptually similar to but physically different from molecular diffusivity (see Appendix B).

(a) Because jets entrain fluid in the surrounding medium, the jet density will vary with distance from the nozzle exit. 
It is possible to illustrate the effect of the diffusion by random motions by performing a simple experiment. Place a horizontal grid near the lower regions of a container filled with a settling slurry and vibrate the grid until the particle distribution is steady. Small scale turbulent motions will cause particles to move randomly throughout the mixture. Because gravity will tend to cause settling, the solids volume fraction decreases with increasing height. The concentration will be found to be a function of a number of parameters including location, Reynolds number, and others $^{(a)}$. That is,

$$
\frac{\overline{\varepsilon_{s}}\left(z^{\prime} d_{g}\right)}{\overline{\varepsilon_{S}}}=f\left(z / d_{g}, \operatorname{Re}_{d g}, F_{g}, \frac{V_{S}}{W_{g}}, \overline{\varepsilon_{s}}, \frac{\rho s}{\rho}, \frac{W_{g}}{\omega d_{g}}\right)
$$

where $\mathbf{z}=$ the elevation above the grid

$d_{g}=$ the grid spacing

$\mathrm{Fr}_{\mathrm{g}}=\frac{\mathrm{W}_{0}{ }^{2}}{\mathrm{~d}_{\mathrm{g}} \mathrm{g}}$, the grid Froude number

$\operatorname{Re}_{\mathrm{dg}}=\frac{\rho \mathrm{W}_{\mathrm{g}} \mathrm{d}_{\mathrm{g}}}{\mu}$, the grid Reynolds number

$\rho_{\mathrm{s}}=$ the density of the solid particles

$\rho=$ the density of the liquid in the slurry

$\overline{\varepsilon_{S}}\left(z / d_{g}\right)=$ the solids volume fraction as a function of elevation above the grid

$V_{S}=$ the unhindered settling velocity of the particles in the slurry

$\mathrm{W}_{\mathrm{g}}=$ the peak velocity of the grid

$\omega=$ circular frequency of the grid motion.

The relevance of this example to jet mixing lies in the similarity of the idealized experiment to random motions imposed by turbulence induced by the jet. At sufficiently high jet Reynolds numbers, flow will be turbulent in some regions of the tank. All turbulent flows will have some measurable characteristic length scale. In the simple example above, the characteristic length scale of the

(a) Those familiar with modeling theories that introduce the concept of "eddy" diffusivity may be puzzled by the absence of the particle response time Stokes number (i.e., the ratio of the particle response time to the time scale of the turbulent fluctuations) in this relation. There is an algebraic constraint between the settling velocity ratio, the particle Stokes number, and the Froude number, i.e., $\tau_{p} / \tau_{1}=V_{S} / W_{g}$ Frg. Consequently, it is appropriate to include only two of the three independent parameters in the functional form; the effect of the third parameter is accounted for automatically. 
turbulence is comparable to the grid spacing, but during jet mixing the characteristic length will vary spatially throughout the tank and will be affected by the size of the tank and size of the jet nozzie. Likewise, the characteristic velocity of the turbulence in the grid experiment is comparable to the grid velocity near the grid but will decay with distance from the grid; during jet mixing, the characteristic velocity will scale with the jet nozzle velocity. In both cases, qualitative features of the turbulence can be characterized using a Reynolds number and a Froude number. Both parameters will be important io the jet mixing problem as well as in the hypothetical experiment posed.

\subsubsection{Dimensionless Parameters Governing Mixing}

It is anticipated that particle transport by large scale motions and by diffusion will occur during hydraulic mitigation efforts. During most proposed applications, transport by diffusion will dominate in some regions and bulk transport will be dominate near the jet nozzle. In some regions, transport by both mechanisms will be comparable.

The possibility that both mechanisms will lead to mixing of solids in SY-101 implies that both must match in the correct proportions if experimental results are to be relevant. It can be argued on the basis of physical understanding that diffusive motions are more strongly infuenced by the Reynolds number and that the influence of gravity on the jet is more strongly influenced by the Froude number and relative density difference of the jet $\mathrm{N}_{\rho}$. However, in the actual application, the relative density of the jet may be affected by the strength of the diffusive motions. Consequently, it is anticipated that variation of each mechanism with Reynolds number and Froude number will almost certainly be nonlinear.

The complicated nature of the interactions between Reynolds number, Froude number, and the density ratio $N_{\rho}$ make it difficult to extrapolate data from one set of Reynolds number, Froude number, and $N_{\rho}$ iu soine other set of values. For example, if two mitigation tests are run in which the Froude and $N_{\rho}$ numbers are matched but Reynolds numbers differ, then the small scale diffusive structure could differ. Positive feedback between the small- and large-scale structures might cause both the large- and small-scale structures to differ. ${ }^{(a)}$

A second example is more relevant to testing hydraulic mitigation strategies in SY-101. If two tests are run in which the Reynolds number and $\mathrm{N}_{\rho}$ match but have different Froude numbers, then (unless there were sufficient small scale turbulence to ensure that the tank was fully mixed) the large scale motion of the jet would differ in both cases. Greater stratification would be expected in the low Froude number case. Because of the specific limitations to scaled testing of mixing technology, scaled tests run at matching Reynolds numbers result in Froude numbers that are larger

(a) For examples of difficulties which arise when experiments are nonsimilar, see Appendix A. 
than would occur in SY-101. Consequently, less stratification would be observed during tests in the scale model tank than would occur in SY-101 at a similar Reynolds number and density ratio $\mathbf{N}_{\rho}$.

Unfortunately, the interaction between large scale and small scale motions makes it impossible to identify a "correction" parameter to account for the lack of similarity between experiments. Consequently, scale-up of data would be problematic because the effects of increased stratification or decreased diffusivity would not be known.

The difficulties involved in scale-up of data could be somewhat minimized if validated numerical cores existed. Unfortunately, more detailed submodels for turbulence, particle motions, and the effect of concentration on viscosity are required to predict a stratified case than to predict an unstratified case. Consequently, care must be taken to perform experiments which fully test all required submodels. This might require a large number of experiments if similarity cannot be achieved.

Because of the inherent limitations, inherent to the scale-up of data without the use of a code and the limitations of any code that has been validated using cases which do not fully exercise all submodels in the code, it is recommended that experiments used to test hydraulic mitigation strategies be designed to reproduce both the correct large scale and small scale mixing mechanisms. This requires that the jet density ratio $\mathrm{N}_{\rho}$, the jet Froude number, and the jet Reynolds number all match those that will occur in the tank as close y as possible. If it is not possible, a sufficient number of cases should be run to allow extrapolation to full scale. 


\subsection{SCALING ANALYSIS OF BEHAVIOR DURING JET MITIGATION EEFORTS}

This section provio'es a mathematical scaling law analysis that results in dimensionles; parameters that govern similarity between scaled experiments and full scale operations. The dimensionless parameters may be manipulated to provide scaling laws for operating parameters, such as nozzle velocities and pump power, used in a scale model test tank and those required to achieve similar results in a full scale tank. These scale-up laws will be applied to obtain algebraic relations which describe the relationship between measured values in a scaled experiment and required values to achieve mitigation in SY-101.

The scaling law analysis is based on the theory of similitude. The theory of similitude is well documented and is introduced in nearly all introductory fluid dynamics or heat transfer texts used at schools with accredited undergraduate programs in chemical, mechanical, or civil engineering; examples include Leinhard (1981), Fox and McDonald (1978), and White (1979). A brief synopsis of the concept of similitude is provided in Section 3.1.

Application of the concept of similitude requires a description of the features of SY-101 contents that are relevant from the fluid dynamics perspective. A brief description of the tank contents is provided in Section 3.2.

Appropriate equations that describe the physical dynamics inside the tank during jet mixing are identified based on the description of the tank contents and on the discussion of the physical mechanisms involved in hydraulic mitigation in Section 2.2. The modeling assumptions made during scaling analysis are in Section 3.2; the final dimensional transport equations describing the tank contents are in Section 3.5.

The governing equations are manipulated into dimensionless form using imposed velocity, length scales, and time scales. The characteristic scales selected to nondimensionalize the transport equations are described in Section 3.6, and the resulting dimensionless equations are in Section 3.7.

Analysis requires preliminary definitions of several variables, which are presented in Section 3.3. The results of the similarity analysis are used to specify the scale-up relations and to determine the appropriate yanperties for dynamic simulants in Section 3.7.(a)

(a) The method presented here is mathematical and relies on use of a mixture model to describe the slurry motion. Similar parameters may be obtained by simple dimensional arguments. Bamberger et al. (1990) presented an analysis of the mixing problem that used the Buckingham Pi method to obtain similarity parameters for mixing; the earlier analysis was designed to study well mixed tanks and dose not include factors that describe the ability of the jet to erode the sludge interface. The parameters obtained in Bamberger et al. are consistent with those obtained by this analysis. The Buckingham $\mathrm{Pi}$ theorem may be used to 


\subsection{CONCEPT OE SIVILITUDE}

When experimental testing of complicated phenomena in a full size prototype is impossible, scaled testing is the only feasible way to determine functional relationships to be used to estimate design parameters. If predictions of prototype behavior are to be based on scaled experiments, it is obvious that the behavior of the prototype and the scale model must be "similar," that is related by clearly identifiable scaling laws. The general conditions necessary to ensure similarity are described in many undergraduate fluid mechanics texts iscluding Fox and McDonald (1978). The conditions required to achieve similarity are described in this section.

Similarity between conditions occurring in an experiment performed in a scaled model and those which occur in a prototype will exist if three conditions are met:

- The scale model and the full scale tank must be geometrically similar, all linear dimensions of the model must be related to the corresponding dimensions of the full scale tank by a constant scale factor.

- The model and the full scale tank must be kinematically similar. The velocities at any two points in the similar tanks must be in the same direction, and all velocities in the model tank will be related to those in the full scale tank by a constant scale factor, all streamlines must be identical.

- The model and the prototype must be dynamically similar; the force distribution at all points in the flow must be scaled linearly between the prototype and the model.

Achieving dynamic similarity is the most restrictive requirement because dynamic similarity can only be achieved when kinematic similarity and geometric similarity are achieved.

In the case of modeling SY-101, geometric similarity requires that the model tank be a cylindrical tank and that the ratio of the level of the tank contents to the diameter of the tank in the model should match this ratio in SY-101. In addition, geometrical features, such as the diameter of nozzles and elevation of the pump above the tank floor, should also be scaled using the same linear scaling factor as the tank diameter and fluid height. That is, if the diameter of the tank is $1 / 12^{\text {th }}$ of the full scale tank, then the nozzle diameter, fluid height, and all other features should be $1 / 12^{\text {th }}$ that of the full scale application. Because the wall will form bounding streamlines and will also impose a no-slip condition on the fluid flow at the wall, kinematic similarity and dynamic similarity

show that numerous sets of similarity parameters exist. Those obtained for this problem will be identical to any obtained using Buckingham $\mathrm{Pi}$ within some degree of algebraic manipulation, provided that all the dimensional properties used in the Buckingham Pi method appear in the equations of motion describing the flow in the tank. For a more complete discussion of the Buckingham Pi method as applied to mixing of a slurries in double-shell tanks, refer to Bamberger et al. 
also require the tank to be geometrically similar. Because bounding streamlines and no-penetration conditions at the wall will affect recirculation patterns in the tank, the scale model tank should have contours similar to those in SY-101.

Once geometric similarity is achieved, then dynamic similarity and kinematic similarity may be achieved by matching all dimensionless parameters which apply to the flow in the tank. The dimensionless parameters can be obtained either by using Buckingham Pi theorem to obtain groups or by identifying a mathematical model that describes the flow field and mathematically manipulating the governing equations into a dimensionless form.

The advantages of the Buckingham Pi method are speed and simplicity. There are two disadvantages to the method: 1) the dimensionless groups obtained are not unique in the sense that any linear algebraic combination of the dimensionless group could also be used to describe the physical phenomena in the applications, ${ }^{(a)}$ and 2) it is possible to overlook parameters that affect the flow field.

(a) This can be illustrated best by an example. Using dimensional arguments alone, it is possible to conclude that the pressure drop per unit length $(\Delta \mathrm{P} / \mathrm{L})$ during flow of a Bingham plastic fluid at a mean velocity $U$ through a pipe with diameter $D$ can be predicted using a functional relation of the form

$$
\mathrm{f}=f(\mathrm{Re}, \mathrm{Y})
$$

where $f=\frac{(\Delta P / L) D}{0.5 \rho U^{2}}$ is the friction factor

$$
\begin{gathered}
\operatorname{Re}=\frac{\rho U D}{\mu} \text { is the Reynolds number } \\
Y=\frac{D \tau_{y}}{U \mu} \text { is the "yield parameter." }
\end{gathered}
$$

The fluid properties for Binham plastic fluid are density, $\rho$, consistency index, $\mu$, and yield stress, $\tau_{\mathrm{y}}$.

However, the functional representation is nonunique because the friction factor may also be expressed in terms of the Reynolds number and the "Hedstrom" number defined as

$$
\mathrm{He}=\frac{D^{2} \tau_{y} \rho}{\mu^{2}}=\operatorname{Re} \mathrm{Y}
$$

Because the Hedstrom number is a product of the Re and $\mathrm{Y}$ numbers, there is no real difference between the statement that the friction factor is a function of the Reynolds and yield numbers and the statement that it is a function of the Reynolds and Hedstrom numbers. Any algebraic relation expressed in terms of $\mathrm{Re}$ and $\mathrm{Y}$ may be algebraically manipulated into a form containing $\mathrm{Re}$ and He. However, the representations appear different, which can be confusing. 
The advantages of scaling the mathematical model are that 1) the most appropriate form of the dimensionless groups will be identificd, and that 2) all dimensional parameters governing the flow field will be automatically identified. Dimensional reasoning can be used to show that dimensionless groups obtained using either method will be identical (within some amount of algebraic manipulation).

\subsection{MATHEMATICAL MODELING ASSUMPTIONS}

This section provides a mathematical model which accounts for the relevant factors affecting the success of hydraulic jets during two stages of the mitigation process: the resuspension of the lower immobilized sludge layer and the mixing period in which solids are maintained in suspension.

The mathematical model is based on a conceptual model of the tank contents and phenomena which are provided below:

- The tank contents are classified into slurry region and a sludge region. (a) $^{(}$

- The sludge region is the settled sludge layer and is defined as a region in which the solid phase has reached the maximum packing fraction possible for the waste material. In this region, the solids do not move relative to the liquid. It is assumed to act as a solid which yields when some critical stress level is exceeded. This critical stress level will be called the "shear strength" and be denoted as $\tau_{\mathrm{ss}} ;$; is is expected to be approximately equal to the magnitude measured using a shear vane.

- The slurry region is defined as a region in which suspended particles in a liquid move relative to the solid phase. When sludge is mobilized by jets, it will disintegrate and mix with liquid, and the mixed material will become slurry.

- The slurry portion of the waste is treated as a continuous non-Newtonian fluid with an effective viscosity that varies as a function of concentration and fluid deformation (or strain rate). A general mixture equation that is applicable to both Newtonian and nonNewtonian slurries will be used during analysis.

- The motion of the particle phase is modeled by a modified diffusion equation that includes the effect of gravity.

(a) Convective and nonconvective regions are now commonly used definitions at Hanford. Sludge and slurry are more useful terms when analyzing mitigation. Slurry and sludge are distinct from convective and nonconvective in the following sense. The convective region is the region in the tank where natural convection induces circulation patterns that prevent large temperature gradients; the nonconvective region is the region in which circulation patterns either do not exist or are too weak to eliminate temperature gradients. It is possible that the nonconvective region is sludge and the convective region is slurry. However, when the jets are activated and mobilization begins, the nonconvective and convective terminology will no longer be useful. Some settled material will mix with supernatant and result in larger amounts of slurry. 


\subsection{PRELIMINARY DEFINITIONS}

Dimensional analysis of the differential equations that would govern the behavior of the tank contents hydraulic during mitigation efforts will be represented by using tensor notation and will use the summation convention. The summation convention is described in Section 3.3.1. The physical parameters required by similarity analysis are described (defined) in Section 3.3.2.

\subsubsection{Indicial Notation and Summation Convention}

Indicial notation allows each component of a vector to be written using the subscript " $\mathrm{i}$ ". A position vector, $x$, may be decomposed into three orthogonal coordinates $x_{1}, x_{2}$, and $x_{3}$, or alternatively as $x_{i}$ with $i$ denoting that it is the "ith" coordinate. Likewise, the velocity may be decomposed into three components with $u_{i}$ denoting the ith velocity component.

Repeated subscripts are used to show which factors are to summed. Thus the quantity $\mathrm{x}_{\mathrm{i}} \mathrm{x}_{\mathrm{i}}=\mathrm{x}_{1} \mathrm{x}_{1}+\mathrm{x}_{2} \mathrm{x}_{2}+\mathrm{x}_{3} \mathrm{x}_{3}$. Similarly, the divergence of a velocity vector is written

$$
\frac{\partial u_{i}}{\partial x_{i}}=\frac{\partial u_{1}}{\partial x_{1}}+\frac{\partial u_{2}}{\partial x_{2}}+\frac{\partial u_{3}}{\partial x_{3}}
$$

When the summation convention is used, a subscript can be repeated two times at most.

\subsubsection{Definition of Physical Parameters}

Solids loading can be described by using solids volume fraction, slurry density, or solids concentration. Definitions for these quantities are provided in this section.

\subsubsection{Definition of Solids Volume Fraction}

A solids volume fraction is defined as the fraction of the total volume of a mixture which is occupied by the solids. The local solids volume fraction, $\varepsilon_{\mathrm{S}}$, is defined as

$$
\varepsilon_{S}(x)=\frac{\text { volume of solids }}{\text { volume of }(\text { solids }+ \text { liquid })}
$$

in a small region surrounding the point $\mathbf{x}$.

The local solids volume fraction varies spatially throughout the tank because solids loading varies throughout the tank; it may also vary temporally when the flow is time dependent.

The mean solids volume fraction characterizes tank contents as a whole; it can be calculated as 


$$
\overline{\varepsilon_{S}}=\int_{\mathcal{V}_{t}} \varepsilon_{\mathrm{S}}(\mathbf{x}) \mathrm{d} \mathcal{V}=\frac{\text { volume of solids in the entire tank }}{\text { volume of (solids + liquid) in the entire tank }}
$$

where $\mathcal{V}_{t}$ is the total volume of waste contained in the tank and is equal to the sum of the volume of solids and the volume of liquid.

The mean solids volume fraction will remain constant during mitigation effort if there are no significant chemical reactions, solids do not dissolve or precipitate, and mitigation does not involve transport to or from the tank.

In principle, the solids volume fraction is bounded by zero and one. A solids volume fraction of zero corresponds to pure liquid, and a solids volume fraction of one corresponds to pure solids. In practice, the solids volume fraction is limited even further, because solids packing has a maximum possible physical level. The maximum achievable solids volume fraction will vary depending on the size distribution of the solids, their shapes, and a number of other factors. The existence of a maximum solids volume fraction imposes a constraint on the solids volume fraction

$$
0<\varepsilon_{\mathrm{S}}<\varepsilon_{\mathrm{S}, \max }
$$

where $\varepsilon_{\mathrm{S}, \max }$ is the maximum possible solids volume fraction of the solids.

\subsubsection{Definitions of Densities}

The definitions of the densities used here assume that the slurry consists of solids and liquid only. The decision to neglect the presence of the sas is based on the assumption that only extremely small amounts of gas can be retained by a mobilized slurry. This assumption, however, must be confirmed.

The local mixture density of a slurry containing solids and liquids only, $\rho_{m}$, depends on the densities of the solids and liquids and on the solids volume fraction

$$
\rho_{m}=\rho_{1}+\left(\rho_{s}-\rho_{1}\right) \varepsilon_{S}
$$

$$
\text { where } \begin{aligned}
\rho_{\mathrm{m}} & =\text { mixture density } \\
\rho_{1} & =\text { liquid or supernatant density } \\
\rho_{\mathrm{s}} & =\text { solids density. }
\end{aligned}
$$


When the tank is fully mixed, the mixture density everywhere in the tank will be equal to the mean mixture density $\overline{p_{m}}$; this may be calculated as

$$
\overline{\rho m}=\rho 1+\left(\rho_{s}-\rho 1\right) \overline{\varepsilon_{s}}
$$

where $\overline{\rho_{m}}$ is the mean mixiure density.

The difference between the local mixture density and the mean mixture density is

$$
\Delta p m=\rho m-\overline{p m}=\Delta p \Delta \varepsilon_{s}
$$

where $\Delta p=p_{S}-p_{1}$ is the density difference between the solids and liquids

$\Delta \varepsilon_{S}=\varepsilon_{S}-\overline{\varepsilon_{S}}$ is the difference between the solids volume fraction at any point in the tank and the mean solids vol'ume fraction for the tank as a whole.

\subsubsection{Definition of Effective Viscosity}

Slurry mixtures may exhibit either simple Newtonian or strain rate dependent non-Newtonian rheology. The mathematical model described in Section 3.4 will assume the possibility of nonNewtonian rheology but can also be applied if the tank contents are Newtonian. Variations of viscosity with temperature are iseglected because large temperature gradients are not anticipated during mitigation. The relevant viscosity information would, however, be the viscosity values at the average tank temperature.

The slurry mixture will be assumed to behave as a fluid with an effective viscosity He. The stress in a fluid may thus be written in the form

$$
\tau_{\mathrm{ij}}=\mu_{\mathrm{e}} \Delta_{\mathrm{ij}}
$$

where

$$
\Delta_{i j}=\left(\frac{\partial w_{i}}{\partial x_{j}}+\frac{\partial w_{j}}{\partial x_{i}}\right)
$$

The quantity $\Delta_{\mathrm{ij}}$ is the slurry strain rate tensor and describes the rate at which the fluid is deformed. 
In a shear layer the strain rate has only one component and the resulting shear stresio is

$$
\tau_{x y}=\mu_{e} \frac{\partial w_{x}}{\partial y}
$$

The variation of the viscosity with concentration and strain rate may be accounted for by assuming that it obeys the following general functional form

$$
\mu_{e}=\mu_{\varepsilon o} f_{\mu}\left(\Delta, \varepsilon_{s}\right)
$$

where $\Delta \quad$ denotes the three invariants of the fluid strain rate tensor collectivily. For a discussion of these invariants see Yih (1977).

$\mu_{e}=$ effective mixture viscosity

$H_{e 0}=$ effective mixture viscosity measured at the mean tank solids volume fraction $\bar{\varepsilon}_{s}$ and the arbitrarily selected reference strain rate, which is $\Delta=W_{0} / H_{t}$

$f_{\mu}\left(\Delta, \varepsilon_{s}\right)=$ a function describing the variation of viscosity with strain rate and concentration.

In the argument above, the general functional form is provided for $f_{\mu}$. This functional form can only be made specific by assuming some type of theology for the slurry mixture. As an example, the form for Bingham plastic is provided below.

For a Bingham plastic, the shear stress tensor in a 1 dimensional flow varies with strain rate as

$$
\tau_{x y}=\tau_{0}\left(\varepsilon_{S}\right)+k\left(\varepsilon_{S}\right) \frac{\partial w_{x}}{\partial y}=\mu_{e o} f_{\mu}\left(\Delta, \varepsilon_{S}\right) \frac{\partial w_{x}}{\partial y}
$$

where $\tau_{0}=$ the yield stress which is assumed to vary with solids volume fraction $\mathbf{k}=$ the consistency index which is also assumed to vary with solids volume fraction.

Algebraic manipulation results in the following functional form:

$$
f_{\mu}\left(\Delta, \varepsilon_{S}\right)=\frac{\tau_{o}\left(\varepsilon_{S}\right)}{\mu_{e o} \partial w_{x} / \partial y}+\frac{k\left(\varepsilon_{S}\right)}{\mu_{e o}}
$$




\subsubsection{Definition of Mean Settling Velocity}

The tendency of particles to settle in the slurry is based on the unhindered settling velocity of particles in the mixture. The effect of variable size distribution is neglected in this analysis; it is assumed that the mean settling velocity may be characterized by the average settling velocity of particles in the mixture. The mean settling velocity of particles in a region of slurry is expected to vary with particle solids volume fraction. This is due in part to the hindering effect of other particles. This variation with loading may be accounted for by assuming that the particle settling velocity obeys a relation of the form

$$
\mathrm{V}_{\mathrm{si}}\left(\varepsilon_{\mathrm{S}}\right)=\mathrm{V}_{\mathrm{soi}} \mathrm{f}_{\mathrm{SO}}\left(\varepsilon_{\mathrm{S}}\right)
$$

where $\mathrm{V}_{\mathrm{si}}=$ the mean particle settling velocity of an individual particle falling through a slurry mixture with concentration $\varepsilon_{S}$

$\mathrm{V}_{\text {soi }}=$ the velocity at which an individual solid waste particle would settle if immersed in a fluid with the reference mixture viscosity $\mu_{e 0}$

$f_{S O}\left(\varepsilon_{s}\right)=a$ dimensionless settling velocity function which describes the variation of settling velocity with solids volume fraction.

For small spherical particles that settle in a fluid with a density and viscosity that matches the density that the tank contents would have when fully mixed, the unhindered settling velocity may be calculated using the Stokes approximation.

$$
V_{\text {soi }}=\frac{\rho_{\mathrm{S}}-\rho_{\mathrm{m}}}{\overline{\rho_{\mathrm{m}}}} \tau_{\mathrm{p}} \mathrm{gi}_{\mathrm{i}}
$$

where the $\tau_{p}$ is defined

$$
\tau_{p}=\frac{2 a^{2}}{9 v_{e}}
$$

where $\mathrm{gi}_{\mathrm{i}}=$ the body force due to gravity

$v_{\mathrm{e}}=\mu_{\mathrm{ed}} / \mathrm{\rho m}_{\mathrm{m}}$ is the reference dynamic viscosity of the mixture.

The irregular shapes of waste particles, the effects of conces ration on settling, and other factors complicate the prediction of the settling velocities of actual waste particles. When possible, 
the actual settling velocities of particles in real waste and in simulated waste should be experimentally determined.

\subsubsection{Definition of Solids Concentration}

It is convenient to use solids concentration during actual specification and manufacture of the simulant properties. Simulants that mimic the physical properties of the contents of SY-101 will eventually be required to conduct scaled experiments. For this reason, this convenient property will be defined here. The solids concentration $c_{s}$ is defined as the mass fraction of solids in a sample:

$$
\mathrm{c}_{\mathrm{s}}=\frac{\text { mass of solids }}{\text { total mass of mixture }}
$$

This may be related to the volume fraction and density ratios as

$$
c_{s}=\frac{\rho_{s} \varepsilon_{s}}{\rho m}
$$

This is algebraically identical to

$$
c_{S}=\left[\frac{\rho l}{\rho_{S}} \varepsilon_{S}^{-1}+\frac{\Delta \rho}{\rho_{S}}\right]^{-1}
$$

Any two simulants with identical solids volume fractions $\varepsilon_{\mathrm{S}}$ and solid to liquid density ratios $p 1 / \rho s$ will also have identical solids concentrations. ${ }^{(a)}$ (This will greatly simplify manufacture of simulants because it is easier to carry out instructions in terms of solids concentrations and density ratios than in terms of the solids volume fraction and density parameters that appear in the governing transport equations in Section 3.5.)

\subsection{MOXTUREMODEL}

The partial differential equation describing the flow are provided in 3.4.1. Boundary conditions are provided in 3.4.2.

(a) Note that two slurries with identical density ratio $\mathrm{\rho l} / \rho_{\mathrm{S}}$ will also have identical magnitudes of the ratio $\Delta \rho / \rho_{S}=1-\rho 1 / \rho_{S}$. 


\subsubsection{Continuity and Momentum Equations}

Transport equations for describing the motion of the slurry are provided in this section. These equations provide a mathematical description of the motion of sludge and slurry in SY-101. The transport equations described here are based on the assumption that mass is conserved in each phase and that Newton's second law des ribes changes in momentum. Conservation of mass and momentum are described by the following equations:

- Continuity equation for the mixture

$$
\frac{\partial \rho_{m}}{\partial t}+\frac{\partial}{\partial x_{i}}\left\{\rho m w_{i}\right\}=0
$$

- Continuity equation for the particle phase

$$
\rho_{s}\left[\frac{\partial \varepsilon_{S}}{\partial t}+\frac{\partial}{\partial x_{i}}\left\{\varepsilon_{s} v_{i}\right\}\right]=0
$$

Adding and subtracting the divergence, $\frac{\partial}{\partial x_{i}}\left\{\varepsilon_{s} w_{i}\right\}$, results in a more convenient form

$$
\rho_{s}\left[\frac{\partial \varepsilon_{S}}{\partial t}+\frac{\partial}{\partial x_{i}}\left\{\varepsilon_{s} w_{i}\right\}+\frac{\partial}{\partial x_{i}}\left\{\varepsilon_{s}\left(v_{i}-w_{i}\right)\right\}\right]=0
$$

- Momentum equations for the mixture

$$
\frac{\partial \rho_{m} w_{i}}{\partial t}+\frac{\partial}{\partial x_{j}}\left[\rho_{m} w_{i} w_{j}\right\}=\frac{\partial}{\partial x_{j}}\left[-P \delta_{i j}+\left\{\mu_{e}\left(\frac{\partial w_{i}}{\partial x_{j}}+\frac{\partial w_{j}}{\partial x_{i}}\right)\right\}\right]+\rho_{m} g_{i}
$$

where $\partial_{\mathrm{ij}}=$ the krokener della. Sha and Soo (1979) propose a similar equation in which the form of the stress tensor is not specified and in which body forces are neglected. It is assumed here that $\tau_{i j}=\mu_{e}\left(\frac{\partial w_{i}}{\partial x_{j}}+\frac{\partial w_{j}}{\partial x_{i}}\right)$ as specified in Equation (3.11) and a body force $\rho_{m} g i$ has been added.

- Particle transport modeled by using a modified diffusion equation

$$
\varepsilon_{S}\left(v_{i}-w_{i}\right)=\varepsilon_{S} V_{s i}-\mathcal{D}_{p} \frac{\partial}{\partial x_{i}} \varepsilon_{S}
$$

where $w_{i}=$ the velocity of the mixture as a whole 


$$
\begin{aligned}
& \mathbf{v}_{\mathbf{i}}=\text { the velocity of the particle phase } \\
& \mathcal{D}_{\mathrm{p}}=\text { the particle diffusivity } \\
& P=\text { the absolute pressure. }
\end{aligned}
$$

The importance of both large-scale motion and small scale fluid motion on particle mixing was emphasized in Section 2.2. Large- scale circulation patterns directly affect the functional form of the velocity field $w_{i}$, which appears on the left side of the particle diffusion equation. Better mixing may be achieved by increasing the average upward component of the mixture velocity $\mathrm{w}_{\mathrm{i}}$ or by increasing the magnitude of the particle diffusivity $\mathcal{D}_{\mathrm{p}}$. In general, increasing the nozzle velocity causes both to occur. The final factor affecting the particle distribution is the particle settling velocity $\mathrm{V}_{\mathrm{si}}$; it is obvious that slurries containing particles that settle rapidly will require more agitation to maintain suspension.

It is possible to eliminate the particle velocity $v_{i}$ as a variable and ivrite Equations 3.19 and 3.21 as a single equation:

$$
\rho_{s}\left[\frac{\partial \varepsilon_{S}}{\partial t}+\frac{\partial}{\partial x_{i}}\left[\varepsilon_{s} w_{i}\right\}+\frac{\partial}{\partial x_{i}}\left\{\varepsilon_{s} V_{s i}\right\}-\frac{\partial}{\partial x_{i}}\left\{\mathcal{D}_{p} \frac{\partial}{\partial x_{i}} \varepsilon_{s}\right\}\right]=0
$$

This set of coupled differential equations govern flow and particle distribution in the tank. Boundary conditions must be specified to completely define the problem.

\subsubsection{Boundary Conditions}

Boundary conditions affect the flow pattern in the tank and may impose their own similarity parameters. The boundary conditions exist at each wall, at the sludge/slurry interface, and at the upper surface of the tank waste; these boundary conditions are described in this section.

\subsubsection{Boundary Condition at Walls}

The no-slip condition governs at any solid surface; consequently, the mixture velocity will be equal to zero at the tank wall.

$$
w_{i}=0
$$




\subsubsection{Boun lary Condition at the Sludge/Slumy Interface}

The sludge/slurry interface is expected to act as a solid when the stress applied at the surface is less than the shear strength of the sludge; when the shear strength is exceeded, the interface is expected to yield. This may be expressed mathematically as

$$
\begin{array}{ll}
w_{i}=0 & \text { when }\left|\tau_{\text {app }}\right|<\tau_{\text {ss }} \\
w_{i} \neq 0 & \text { when }\left|\tau_{\text {app }}\right|>\tau_{\text {ss }}
\end{array}
$$

The general relation describing applied stress tensor in a mixture was provided in the definition of the effective viscosity. This may be evaluated at the wall resulting in

$$
\tau_{a p p}=\mu_{e}\left(\frac{\partial w_{i}}{\partial x_{j}}+\frac{\partial w_{j}}{\partial x_{i}}\right)
$$

Similarity analysis may be performed successfully using this representation of the shear stress on the fluid side of the slurry-sludge interface.

\subsubsection{Boundary Condition at the Upper Surface}

The appropriate boundary condition at the upper surface depends on the presence or absence of a crust. Both cases are considered for completeness.

When there is no crust, the upper surface of the slurry is a free surface and is held at a constant pressure equal to the head space pressure. The surface may deform and ripple as a result of fluid motion. Because the surface is a streamline, the relationship between elevation of the free surface, the velocity of the mixture at the surface, and the pressure at the surface is

$$
P_{\mathrm{hs}}+\frac{1}{2} \rho_{\mathrm{m}} \mathrm{wiw}_{\mathrm{i}}+\rho_{\mathrm{mgix}} \mathrm{x}_{\mathrm{si}}=\text { constant }
$$

where $P_{\mathrm{hs}}=$ the pressure in the head space

$x_{s i}=a$ coordinate location of a point on the slurry surface.

This may be written in a more familiar form as Bernouilli's equation 


$$
P_{\mathrm{hs}}+\frac{1}{2} \rho_{\mathrm{m}}|\mathrm{w}|^{2}+\rho_{\mathrm{m}} \mid g / \mathrm{h}_{\mathrm{s}}=\text { constant }
$$

where $h_{s}$ is the distance from the tank bottom to the upper waste surface.

When there is no rippling or surface distortion, the distance from the bottom of the tank, $\mathrm{h}_{\mathbf{s}}$, is a constant. In contrast, when th: are is rippling, the distance from the surface to the tank bottom $\mathrm{h}_{\mathbf{S}}$ may vary spatially.

When a crust, or nondeforming layer, is present, the no-slip condition is imposed and the boundary condition is

$$
w_{i}=0
$$

\subsection{CHARACTERISTIC VELOCITIES, LENGTH SCALES, AND TIME SCALES}

Characteristic velocities, lengths scales, and time scales are used to describe the scale of any physical phenomena and can be used for purposes of dimensional analysis.

\subsubsection{Length Scales}

A length scale describing the tank size must be selected. When experiments are performed in geometrically similar tanks, the characteristic length may be arbitrarily selected to be the depth of the tank contents $H_{t}$, the tank diameter $D_{t}$, the nozzle diameter $D_{0}$, the cube root of the tank volume, or any other geometric feature of interest. The choice is arbitrary if those aspect ratios (such as that of the tanks diameter to the depth of the tank contents $\mathrm{D}_{\rho} / \mathrm{H}_{\mathrm{t}}$ or the nozzle diameter to the depth of the tank contents $D_{0} / H_{\mathcal{V}}$ are identical in both tanks. For the purposes of analysis, it is assumed that experiments will be performed in geometrically similar tanks. The characteristic length selected for the purpose of analysis will be the tank height $\mathrm{H}_{t}$.

Dimensionless coordinates are obtained by taking the ratio of the dimensional coordinate to the characteristic length. Thus

$$
\tilde{\mathbf{x}}_{\mathbf{i}}=\mathbf{x}_{\mathbf{i}} / \mathrm{H}_{\mathbf{t}}
$$

where $\mathrm{H}_{\mathfrak{t}}=$ the depth of the tank contents

$\tilde{\mathbf{x}_{\mathbf{i}}}=\mathbf{a}$ dimensionless coordinate. 


\section{Dimensionless lengths include}

- the dimensionless nozzle diameter, $\tilde{D}_{0}=D_{0} / H_{t}$

- the dimensionless tank diameter, $\tilde{D}_{t}=D_{t} / H_{t}$

- the dimensionless tank height, $\tilde{H}_{t}=H_{t} / H_{t}=1$, which by definition is equal to one.

Geometric similarity requires that the dimensionless nozzle diameter, the dimensionless tank diameter, and any other geometric features be identical in the scale model tank and in implementation in SY-101.

\subsubsection{Characteristic Velocities}

When the jets are activated, the tank contents will be set into motion by jets with a known jet nozzle velocity $W_{0}{ }^{\text {(a) }}$ Velocities in the tank will be scaled using this characteristic velocity. There are three dimensionless velocities of interest defined as follows:

- the dimensionless mixture velocity, which varies throughout the tank

$$
\tilde{w}_{\mathrm{i}}=\mathrm{w}_{\mathrm{i}} / \mathrm{w}_{\mathrm{O}}
$$

- the dimensionless particle velocity, which also varies throughout the tank

$$
\tilde{v}_{i}=\frac{v_{i}}{W_{0}}
$$

- the dimensionless particle settling velocity, which describes the tendency of particles to settle

$$
\tilde{\mathrm{V}}_{\mathrm{si}}=\mathrm{V}_{\mathrm{si}} / \mathrm{W}_{\mathrm{o}}
$$

where $W_{0}=$ the velocity of the mixture at the nozzle

$$
\begin{aligned}
\tilde{v}_{\mathbf{i}} & =\text { the dimensionless particle velocity } \\
\tilde{v}_{\mathbf{s i}} & =\text { the dimensionless settling velocity } \\
\tilde{w}_{\mathbf{i}} & =\text { the dimensionless velocity of the mixture. }
\end{aligned}
$$

Substituting the functional form of the dimensionless settling velocity allows it to be written in the following form:

$$
\tilde{\nabla}_{\text {si }}=\frac{V_{\text {soi }}}{W_{0}} f_{\text {so }}\left(\varepsilon_{S}\right)=\tilde{V}_{\text {soi }} f_{\text {so }}\left(\varepsilon_{S}\right)
$$

(a) The characteristic velocity $W_{0}$ is directly related to the volumetric flow rate $Q_{0}$ through the nozzle with $\mathrm{W}_{0}=4 \mathrm{Q}_{0} \pi \mathrm{D}_{0}{ }^{2}$. 


\subsubsection{Characteristic Pressure}

Pressure will be made dimensionless by using twice the dynamic pressure of the jet:

$$
\tilde{P}=P /\left(\overline{\rho_{\mathrm{m}}} \mathrm{W}_{0}^{2}\right)
$$

The gravitational acceleration vector will be made dimensionless by using the magnitude of the gravitational acceleration on earth $|g|$, which is equal to approximately $9.8 \mathrm{~m} / \mathrm{s}^{2}$ on earth:

$$
\tilde{g}_{i}=g_{i} / g \mid
$$

\subsubsection{Characteristic Time Scale}

Many of the proposed mitigation methods are inherently unsteady, with some characteristic time scale imposed. The characteristic time scale will be denoted as $\mathrm{T}_{\Omega}$ for all cases. The exact meaning of the time scale will vary from technology to technology. For oscillating jets, the characteristic time scale $T_{0}$ is the period of one jet oscillation; for jets operated using a liquid piston, the characteristic time scale is equal to one piston cycle:

$$
\tilde{t}=t / T_{0}
$$

\subsection{GOVERNING DIFFERENTIAL EOUATIONS IN DIMENSIONLESS FORM}

In this section the characteristic scales defined in Section 3.5 are applied to obtain the dimensionless differential equations. The dimensionless continuity and momentum equations are obtained in Section 3.6.1; the dimensionless boundary conditions are obtained in Section 3.6.2.

\subsubsection{Dimensionless Continuity and Momentum Equations}

Application of the length, time, and velocity scales to the mixture continuity equation (Eq. 3.19) results in

$$
\eta_{\mathrm{t}} \frac{\partial}{\partial \tilde{\mathrm{t}}}\left(1+\mathrm{N}_{\rho} \Delta \varepsilon_{\mathrm{s}}\right)+\frac{\partial}{\partial \tilde{x}_{i}}\left\{\left(1+N_{\rho} \Delta \varepsilon_{s}\right) \tilde{w_{i}}\right\}=0
$$


where $\eta_{t}=\frac{H_{t}}{T_{0} W_{0}} \quad$ a dimensionless time parameter

$$
\mathrm{N}_{\rho}=\Delta \rho / \overline{\rho_{\mathrm{m}}} \begin{aligned}
& \text { a dimensionless density difference between the solids and the } \\
& \text { supernatant. }
\end{aligned}
$$

Two independent parameters appear in this equation; these are the dimensionless time parameter $\eta_{t}$ and the density ratio $\mathrm{N} \rho$. Both will appear in later equations. The dimensionless time parameter describes the effect of the time varying nozzle velocity or its location in the flow. The density ratio describes the importance of the concentration variations to the continuity equation.

Examination of the dimensionless continuity equation (Eq. 3.40) indicates that slurries with different density parameters $\mathrm{N}_{\rho}$ will behave differently. When the density parameter is large, even slight spatial variations in the solids volume fraction will result in large differences in the mixture density. In contrast, when the density parameter is zero, large variations in the solids volume fraction will not result in density difference. This can have an important influence on turbulence characteristics and consequently on solids mixing.

The particle diffusion equation (Eq. 3.24) may be written in dimensionless form as

$$
\begin{aligned}
& \eta_{\mathrm{t}} \frac{\partial}{\partial \tilde{\mathrm{t}}}\left(\overline{\varepsilon_{\mathrm{s}}}+\Delta \varepsilon_{\mathrm{s}}\right)+\frac{\partial}{\partial \tilde{\mathrm{x}}_{\mathrm{i}}}\left\{\left(\overline{\varepsilon_{\mathrm{s}}}+\Delta \varepsilon_{\mathrm{S}}\right) \tilde{\mathrm{w}_{\mathrm{i}}}\right\} \\
& +\mathrm{f}_{\text {so }} \frac{\partial}{\partial \tilde{\mathrm{x}}_{\mathbf{i}}}\left\{\left(\overline{\varepsilon_{\mathrm{S}}}+\Delta \varepsilon_{\mathrm{S}}\right) \tilde{\mathrm{V}}_{\text {soi }}\right\}-\frac{\partial}{\partial \tilde{\mathbf{x}}_{\mathbf{i}}}\left\{\frac{\mathcal{D}_{\mathrm{p}}}{\mathrm{W}_{\mathrm{o}} \mathrm{L}_{\mathbf{0}}} \frac{\partial}{\partial \tilde{\mathrm{x}}_{\mathbf{i}}}\left(\Delta \varepsilon_{\mathrm{s}}\right)\right\}=0
\end{aligned}
$$

Four independently adjustable dimensionless parameters appear in the particle diffusion equation. These are 1) the dimensionless time parameter $\eta_{t}$, which also appeared in the fluid continuity equation; 2) the mean volume concentration $\overline{\varepsilon_{s}} ; 3$ ) the ratio of the characteristic settling velocity of the particles to the jet nozzle velocity $\widetilde{V}_{\text {soi; }}$ and 4 ) the dimensionless function describing the effects of concentration on the settling velocity of individual particles $\mathrm{f}_{\mathrm{so}}$.

The mixture momentum equation (Eq. 3.22) may be written as

$$
\begin{aligned}
& \eta_{t} \frac{\partial}{\partial \tilde{t}}\left\{\left(1+N_{\rho} \Delta \varepsilon_{s}\right) \tilde{w}_{i}\right\}+\frac{\partial}{\partial \tilde{x}_{j}}\left\{\left(1+N_{\rho} \Delta \varepsilon_{S}\right) \tilde{w}_{i} \tilde{w}_{j}\right\} \\
& =-\frac{\partial \widetilde{P}}{\partial \tilde{x}_{i}}+\operatorname{Re}_{H^{-1}}\left\{f_{\mu}\left(\frac{\partial \tilde{w}_{i}}{\partial \tilde{x}_{j}}+\frac{\partial \tilde{w}_{j}}{\partial \tilde{x}_{i}}\right)\right\}+N_{\rho} \operatorname{Fr}^{-2} \tilde{\mathrm{g}}_{i} \Delta \varepsilon_{S}
\end{aligned}
$$


where $R_{\mathrm{H}}=\frac{\overline{\rho_{\mathrm{m}}} \mathrm{W}_{0} \mathrm{H}_{\mathrm{t}}}{\mu_{\mathrm{eo}}}$

$$
\mathrm{Fr}^{2}=\frac{\mathrm{W}_{0}^{2}}{|\mathrm{~g}| \mathrm{H}_{\mathrm{t}}}
$$

To emphasize the importance of density differences, the pressure has been adjusted to account for the mean hydrostatic variation ${ }^{(a)}$ in the tank using

$$
P=P-\overline{\rho_{m}} g_{i} x_{i}
$$

Three new independent parameters appear in this equation:

- the dimensionless function describing the variation of the viscosity with density and shear rate, $\mathrm{f}_{\mu}$

- the tank Reynolds number based on the level of the tank contents, $\operatorname{Re}_{H^{(b)}}$

- the Froude number based on the level of the tank contents, $\mathrm{Fr}^{2}$.

The functional form of the viscosity variation may be expressed in terms of the dimensionless strain rate and the mean solids volume fraction as

$$
f_{\mu}=f_{\mu}\left(\tilde{\Delta}, \overline{\varepsilon_{s}}+\Delta \varepsilon_{s}\right)
$$

(a) The absolute pressure is relevant during analysis involving expansion and compression of a gas, such as during modeling of tank overturm phenomena. However, in flow analysis it is convenient to eliminate the hydrostatic pressure differences that do not result in motion.

(b) The tank Reynolds number based on level of the tank contents describes the degree of turbulence in the tank as a whole. However, the Reynolds number based on the nozzle diameter is more useful for describing the degree of turbulence in the jet itself and for describing the jet motion in general. The Reynolds number based on jet nozzle diameter is

$$
\mathrm{R}_{\mathrm{e}} \mathrm{D}_{\mathrm{o}}=\rho_{\mathrm{m}} \mathrm{W}_{\mathrm{o}} \mathrm{D}_{\mathrm{d}} / \mu_{\mathrm{eo}}=\left(\mathrm{D}_{\mathrm{o}} / \mathrm{H}_{\mathrm{t}}\right) \mathrm{Re}_{\mathrm{H}}
$$

The ratio of the nozzle diameter to the tank height is a constant of geometrically scaled tanks. Consequently, whenever the two tanks are geometrically similar, both Reynolds numbers will match in the full scale and small scale tanks, or neither will. Consequently, the choice between the Reynolds number based on the level of the tank contents or the nozzle diameter is arbitrary for the purpose of designing this particular experiment. 
where the dimensionless strain rate is

$$
\tilde{\Delta}=\frac{\Delta H_{t}}{w_{0}}=\sqrt{\left(\frac{\partial \tilde{w}_{i}}{\partial \tilde{x}_{j}}+\frac{\partial \tilde{w}_{j}}{\partial \tilde{x}_{i}}\right)\left(\frac{\partial \tilde{w}_{i}}{\partial \tilde{x}_{j}}+\frac{\partial \tilde{w}_{j}}{\partial \tilde{x}_{i}}\right)}
$$

The reference viscosity $\mu_{\text {eo }}$ will be the viscosity measured at $\widetilde{\Delta}=1$ and $\varepsilon_{S}=\overline{\varepsilon_{S}}$; this requires the function $f_{\mu}$ to obey $f_{\mu}=1$ when $\tilde{\Delta}=1$ and $\varepsilon_{s}=\overline{\varepsilon_{s}}$.

The Froude number $\mathrm{Fr}^{2}$ describes the ability of the jet to carry solids to the upper regions of the tank. The importance of the densimetric Froude number $\mathrm{Fr}^{2} / \mathrm{N}_{\rho}$ to the mixing times achieved in tanks was identified by Fossett et al. (1949) and Fossett and Prosser (1951) during an experiment designed to study the effect of the Reynolds number on mixing using jets. Qualitative observations indicated that both the jet density ratio $\frac{\rho_{j}-\rho_{m}}{\rho_{m}}$ and the densimetric Froude number have a dramatic effect on the motion of a jet. When the Froude number of a dense jet was low, the jet could not rise to upper regions of the tank. In addition, measurements of the mixing time indicated that the densimetric Froude number was the most important parameter affecting mixing times achieved. Fossett and Prosser's data indicate that an upward facing jet with a density that exceeds the density of the surrounding medium by $1 \%$ required a Froude number of 24 to reach the upper regions of the tank. Horizontal jets will require larger Froude numbers to reach the upper regions of the tank. (a)

\subsubsection{Dimensionless Boundary Conditions}

The boundary conditions may also be may also be expressed in dimensionless form; only two boundary conditions provide additional dimensionless parameters which describe the flow. These are the boundary condition on the upper free surface and the boundary condition at the sludge-slurry interface.

(a) The densimetric Froude number defined by Fosset and Prosser (1951) is

$$
\mathrm{Fr}^{2}=\rho \mathrm{W}_{0}^{2} \sin ^{2}\left(\phi+5^{\circ}\right) / \Delta \rho \mathrm{H}|\mathrm{g}|
$$

where $\rho$ is the density of the surrounding fluid and $\Delta \rho$ is the density difference between the fluid in the jet and the surrounding fluid and $\phi$ is the angle between the jet axis and the horizontal plane. It corresponds closely to the product of the densimetric Froude number and the void deviation $\Delta \varepsilon_{S}$ defined here. 


\section{Upper Surface}

A new dimensionless parameter is introduced when the upper surface is a free surface.

When a crust is present, no new dimensionless parameter is introduced. In this case, the boundary conditions is

$$
\tilde{w}_{\mathbf{i}}=0
$$

No dimensionless parameter appears in this relation.

In contrast, when there is no crust at the upper surface, the dimensionless boundary condition is

$$
\operatorname{Fr}^{2}\left\{\frac{1}{2} \tilde{w}_{i} \tilde{w}_{i}\right\}+\tilde{g_{i}} \tilde{x}_{i}=\text { constant }
$$

Thus, if no crust is present at the top of the tank, the Froude number also governs the degree of rippling at the surface.

\section{Sludge-Slurry Interface}

The boundary condition at the sludge-slurry interface is complicated; three relations were suggested for the form of the shear stress at the boundary. The dimensionless boundary condition becomes

$$
\tilde{\mathbf{w}}_{\mathbf{i}}=0 \quad \text { when }\left|\tau_{\mathrm{app}}\right| / \tau_{\mathrm{ss}}<1
$$

and

$$
\tilde{w_{i}} \neq 0 \quad \text { when }\left|\tau_{\text {app }}\right| / \tau_{\mathrm{ss}}>1
$$

The dimensionless applied shear stress $\left|\tau_{\text {app }}\right| / \tau_{\text {ss }}$ governs the ability of the sludge bank to yield. This applied shear stress is a function of the flow field; the functional form of this stress mist be manipulated to allow identification of an independently adjustable parameter. 
The dimensionless form for the applied shear stress is

$$
\left|\tau_{\text {app }}\right| / \tau_{\text {ss }}=\frac{\overline{\rho_{m}} W_{o}^{2}}{\tau_{s s}} \operatorname{Re}_{H}^{-1} f_{\mu}\left\{\frac{\partial \tilde{w}_{i}}{\partial \tilde{x}_{j}}+\frac{\partial \tilde{w}_{j}}{\partial \tilde{x_{i}}}\right\}
$$

This introduces the final dimensionless parameter, $\frac{\overline{\rho_{m}} W_{0}^{2}}{\tau_{s s}}$, which is the ratio of the dynamic pressure of the jet to the shear stress of the sludge. The functional form of the slurry viscosity $f_{\mu}$ also affects the tendency of the slurry to yield.

\subsubsection{Summary of Dimensionless Parameters}

The previous similarity analysis indicates that conditions in the tank will be a function of the nine similarity parameters. A summary of the nine dimensionless parameters appe uring in ali dimensionless equations is provided in Table 3.1. 
TABLE 3.1. Summary of Dimensionless Parameters

\begin{tabular}{|c|c|}
\hline $\begin{array}{l}\text { Inverse time parameter: } \\
\text { Describes the effect of the time dependence of the } \\
\text { jer on the behavior of the tank contents. }\end{array}$ & $\eta_{t}=\frac{H_{0}}{T_{0} U_{0}}$ \\
\hline $\begin{array}{l}\text { Reynolds number based on tank height: } \\
\text { Describes the degree of turbulence in the tank. }\end{array}$ & $\mathrm{ReH}_{\mathrm{H}}=\frac{\overline{\mathrm{pm}_{\mathrm{m}} \mathrm{W}_{\mathrm{o}} \mathrm{H}_{\mathrm{O}}}}{\mu_{\mathrm{eO}}}$ \\
\hline $\begin{array}{l}\text { Froude uumber: } \\
\text { Describes the general effect of density differences } \\
\text { on the jet motion when density differences exist in the } \\
\text { tank. } \\
\text { Also describes the degree of surface rippling if the } \\
\text { crust absent. }\end{array}$ & $\mathrm{Fr}^{2}=\frac{\left|\mathrm{W}_{\mathrm{O}}\right|^{2}}{\mathrm{gH}_{\mathrm{O}}}$ \\
\hline $\begin{array}{l}\text { Stress ratio: } \\
\text { Describes the ability of the jet to erode settled } \\
\text { slurry. }\end{array}$ & $\mathrm{N}_{\tau}=\frac{\overline{\rho_{\mathrm{m}}} \mathrm{W}_{\mathrm{o}}^{2}}{\tau_{\mathrm{s}}}$ \\
\hline $\begin{array}{l}\text { Settling Velocity ratio: } \\
\text { Describes the tendency of particles to settle. }\end{array}$ & $\tilde{V}_{\text {soi }}=\frac{V_{\text {soi }}}{W_{\text {o }}}$ \\
\hline $\begin{array}{l}\text { Density ratio: } \\
\text { De: cribes the importance of density differences } \\
\text { between the solids and the mixture as a whole. } \\
\text { Appears in both mixture continuity and momentum } \\
\text { equations. }\end{array}$ & $N_{p}=\frac{\Delta \rho}{\overline{\rho m}}$ \\
\hline $\begin{array}{l}\text { Mean solids volume fraction: } \\
\text { Influences the possible magnitude of density } \\
\text { variations in the tank. }\end{array}$ & $\overline{\varepsilon_{S}}$ \\
\hline $\begin{array}{l}\text { Functional variation of settling velocity with } \\
\text { concentration: } \\
\text { Modifies the effect of the settling velocity ratio. }\end{array}$ & $\mathrm{f}_{\mathrm{SO}}\left(\boldsymbol{\varepsilon}_{\mathrm{S}}\right)$ \\
\hline $\begin{array}{l}\text { Functional dependence of viscosity: } \\
\text { Modifies the effect of the Reynolds number. }\end{array}$ & $f_{\mu}\left(\tilde{\Delta}, \overline{\varepsilon_{S}}+\Delta \varepsilon_{S}\right)$ \\
\hline
\end{tabular}




\subsection{EXPERIMENT DESIGN}

In this section, the similarity analyses performed in Sections 3.0 through 3.6 are applied to designing scaled experiments. Goals of these experiments and the use of similarity to design the experiments are described in Section 4.1. Some estimates of the properties in SY-101 are in Section 4.2. A general discussion of methods to achieve similarity are provided in Section 4.2. Specific algebraic relationships for properties are provided in Section 4.2.1; a possible method of performing an experiment at $1 / 12^{\text {th }}$ scale is discussed in Section 4.2.2.

\subsection{GENERALDISCUSSION OF EXPERIMENTALDESIGN}

The goal of scaled experiments using physical simulants is to determine the minimum jet nozzle velocity required $\mathrm{W}_{\text {omin }}$ to first suspend and then to maintain solids in suspension using a particular hydraulic mitigation technology. This may be accomplished by using the scaling law analysis to design the scaled experiments.

Flow in both SY-101 and in the scale model tank is a function of all physical properties, (solid density, liquid density, mixture viscosity, etc.), all geometric features (tank height, nozzle diameter, etc.), and all characteristic velocities and times which appeared in the model equations discussed in Sections 3.0 through 3.6. However, flow in the tank can also be characterized by the eight dimensionless parameters in Table 3.1. The theory of similarity ensures that the degree of suspension achieved in a small-scale tank at specific magnitudes of these eight dimensionless parameters will be identical to the degree of suspension achieved in SY-101 when the same magnitude of these dimensionless parameters is achieved.

For example, if the total fraction of the tank bottom cleared $\widetilde{A}_{c}$ by jets will obey the relation

$$
\tilde{\mathrm{A}}_{c, X}=\tilde{\mathrm{A}}_{c, S Y}
$$

where $\widetilde{A}_{c, X}$ is the ratio of the cleaned sludge area to the total floor area in the scale model tank and $\widetilde{A}_{c, S Y}$ is the ratio of the cleaned sludge area to the total floor area in SY-101, provided that all of the following requirements are met. Then 


$$
\begin{aligned}
& \eta_{\mathrm{t}, \mathrm{X}}=\eta_{\mathrm{t}, \mathrm{SY}} \\
& \operatorname{Re}_{\mathrm{H}, \mathrm{X}}=\mathrm{Re}_{\mathrm{H}, \mathrm{SY}} \\
& \mathrm{Fr}, \mathrm{X}^{2}=\mathrm{Fr}, \mathrm{SY}^{2} \\
& \mathbf{N}_{\tau, \mathbf{X}}=\mathbf{N}_{\mathcal{\tau}, \mathbf{S Y}} \\
& \tilde{\mathrm{V}}_{\text {soi, } X}=\tilde{\mathrm{V}}_{\text {soi,SY }} \\
& \mathbf{N}_{\rho, X}=\mathbf{N}_{\rho S Y} \\
& \overline{\varepsilon_{S}, X}=\overline{\varepsilon_{S}, S Y} \\
& f_{S O, X}=f_{S O, S Y} \\
& f_{\mu}\left(\tilde{\Delta}, \overline{\varepsilon_{s}}+\Delta \varepsilon_{s}\right), X=f_{\mu}\left(\tilde{\Delta}, \overline{\varepsilon_{S}}+\Delta \varepsilon_{S}\right), S Y
\end{aligned}
$$

No statements may be made regarding the relationship between the ratio of the cleaned areas in both tanks if the similarity criteria Equations (4.2-4.10) are not matched. (See Appendix A for an example illustrating the effects of not matching scaling parameters.) It may be possible to relax some similarity criteria on the basis of physical understanding or experimental evidence. However, this must be left to the designers of the scaled experiments because it requires significant technical judgment to identify and justify specific reasons for relaxing each criteria.

A possible procedure for identifying the minimum nozzle velocity required to prevent accumulation of solids may be determined by filling the scale-model tank with an appropriate simulant and increasing the velocity until the condition $\widetilde{\mathrm{A}}_{\mathrm{c}, \mathrm{X}}=0$ is achieved. The minimum velocity required in SY-101 may then be determined by determining the velocity which results in the same magnitude of dimensionless parameters in SY-101.

The procedure for designing an experiment to determine the minimum nozzle velocity is

- Determine the possible magnitude of the independent dimensionless parameters in SY-101.

- Determine the simulant properties which will allow the same range of dimensionless parameters to be achieved in a scaled experiment.

- Select a suitable range of simulant properties for a particular experimental scale.

- Select a range of nozzle velocities of testing.

Tests would be performed at increasing nozzle exit velocities until the minimum velocity required to clear the entire tank bottom was determined. 


\subsection{PROPERTIES OF WASTE CONTAINED IN SY-101}

This section discusses the physical properties of the waste in SY-101 and the possible magnitude of the 10 dimensionless parameters governing behavior of the tank contents during jet mixing.

Properties of the waste in SY-101 that can be dir(xctly related to dimensionless parameters are 1) the mean solids void fraction $\varepsilon_{s} ; 2$ ) the density ratio $N_{p} ; 3$ ) the form of the viscosity variation with concentration and solids volume fraction $f_{\mu}\left(\Delta, \varepsilon_{s}\right)$; and 4$)$ the form of the settling velocity variation with solids volume fraction $f_{s o}\left(\varepsilon_{S}\right)$. These will be discussed first. The discussion of other properties, such as the mixture viscosity, mixture density, and sludge yield stress, are at the end of this section.

Because core sample analyses are ongoing, this section is necessarily incomplete. Further evaluation of the properties in SY-101 are needed for complete definitions of the simulant properties required during scaled testing.

\subsubsection{Solids Volume Eraction}

More information about the properties in SY-101 is needed for complete definitions of the simulant properties to be used in scaled testing. The solids volume fraction in SY-101 as a whole is unknown. It could only be determined from analyses of samples of all core segments from the bottom to the top of the tank; these are not available. In addition, the solids volume fraction is measured indirectly by the volume of settled solids, the volume of centrifuged solids, and the \% weight of water.

Although the data from the individual samples are insufficient to determine the solids volume fraction, the solids volume fraction may be bounded by the values available. Because of physical packing limitations, the solids volume fraction must be less than the volume of centrifuged solids. The maximum solids volume fraction possible for uniformly sized spheres is $74 \%$; this corresponds to a closely packed structure. The maximum packing fraction for tank wastes may be higher or lower. Larger packing fractions could result if the solids consist of nonuniformly sized particles. Alternatively, repulsive forces between solids could result in lower packing fractions.

Table 4.1 presents an analyses of segments 8 and 19 of core sample \#22 from SY-101 reported by Tingey. (a) The density of the sample, the volume of centrifuged solids, and the $\%$ weight of water at $65^{\circ} \mathrm{C}$ are provided in Table 4.1. Table 4.1 also shows an estimate of a solids

(a) Tingey, J. M. 1992. Physical Characterization of Tank 101-SY Core Samples from Window C. PNL-8054, Pacific Northwest Laboratory, Richland. Unpublished. Cited hereafter as Tingey (1992 unpublished report). 


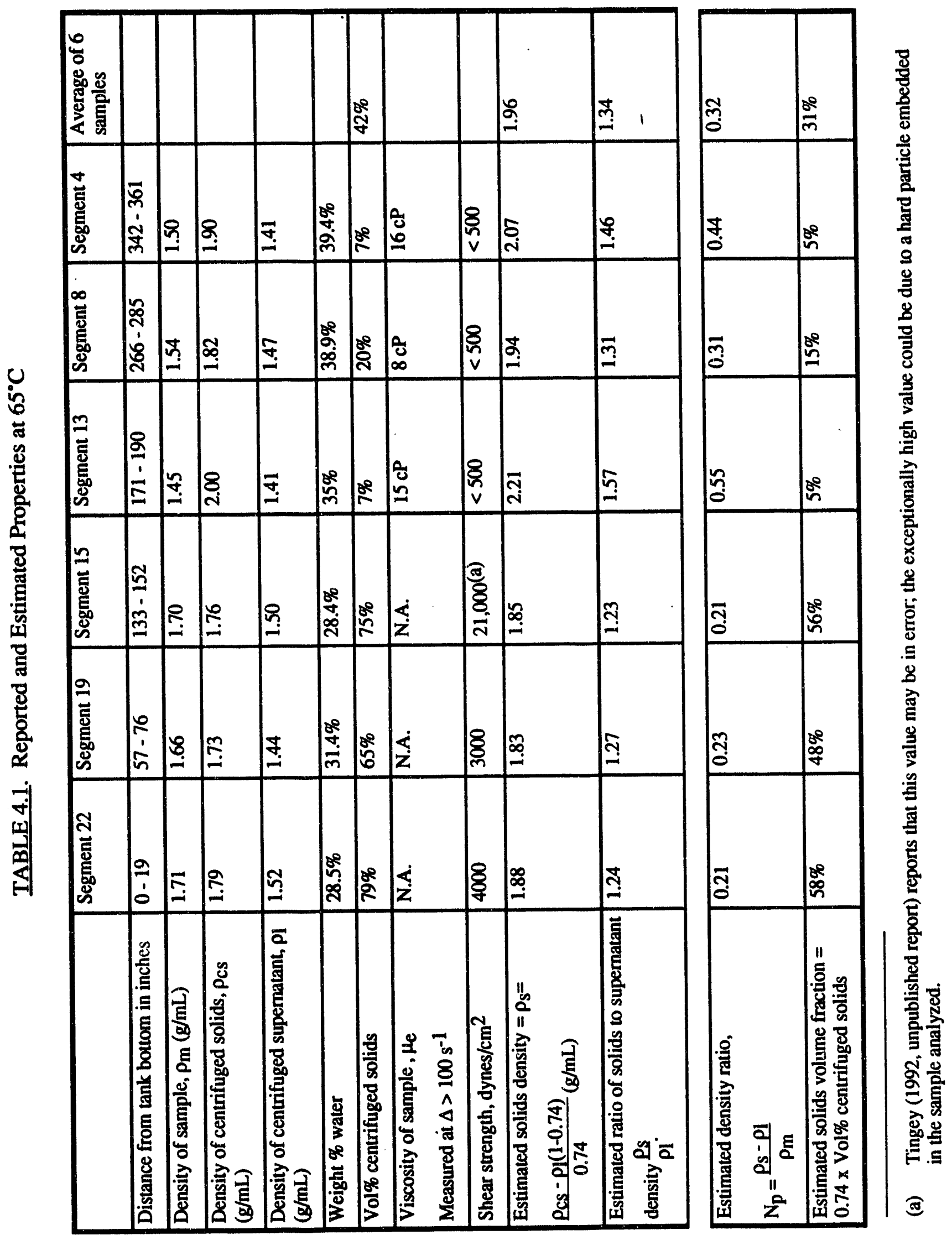


volume fraction for the entire tank assuming that the solids volume fraction is equal to $74 \%$ after centrifuging. The estimated average solids volume fraction based on all six samples is $31 \%$; actual solids volume fractions may be larger or smaller.

\subsubsection{The Density Ratio}

The density ratio of the solids to the mixture density is also not available. Tingey(a) measured and reported the density of the centrifuged supernatant and the centrifuged solids. This information would be sufficient if 1 ) the solids volume fraction of the centrifuged solids is known and 2 ) the density of the interstitial liquid is known. If the solids volume fraction is assumed to be $\varepsilon_{\text {smax }}=74 \%$ and the density of the interstitial liquid is assumed to be equal to the density of the centrifuged supernatant, the solids density is equal to

$$
\rho_{s}=\frac{\rho_{c s}-\rho_{1}\left(1-\varepsilon_{\text {Smax }}\right)}{\varepsilon_{\text {smax }}}=\frac{\rho_{c s}-\rho_{1}(1-0.74)}{0.74}
$$

where $\rho_{c s}=$ the density of the centrifuged solids

$\rho l=$ the density of the liquid or supernatant.

Based on this estimate, the average solids density is $1.96 \mathrm{~g} / \mathrm{mL}$. The maximum estimate is $2.21 \mathrm{~g} / \mathrm{mL}$; the minimum is $1.83 \mathrm{~g} / \mathrm{mL}$. The variation in the estimate could arise from at least two sources. First, it is possible that the average solids density actually varies from region to region in the tank. This could occur as a result of differential settling of more dense or less dense solid constituents. It is also possible that the variation in the estimated solids density occurs because the solids volume fraction in the centrifuged solids may not be equal to 0.74 . No actual data on the solids volume fraction of the centrifuged solids is available; it is unlikely that this value can be determined because of difficulties associated with measuring solids densities of actual samples.

The density ratio is then defined as

$$
\mathrm{N}_{\rho}=\frac{(\rho \mathrm{l}-\rho \mathrm{s})}{\rho_{\mathrm{m}}}
$$

The estimated magnitude of the average density ratio as based on each of the six samples is provided in Table 4.1. The average of the six samples is 0.32 .

(a) Tingey (1992, unpublished report). 


\subsubsection{Viscosity}

The viscosities for segments 4, 8, and 13 were reported by Tingey (1992, unpublished report) who reports that samples appear to be Newtonian at shear rates above $100 \mathrm{~s}^{-1}$. The viscosity of the more concentrated samples taken from the lower nonconvective region were not available as of February 1992.

The magnitude of the sample viscosity at $65^{\circ} \mathrm{C}$ is provided in Table 4.1 .

\subsubsection{Particle Settling Velocity}

Particle settling velocity is extremely difficult to measure from core samples. The closest comparable measurement is the settling velocity of the supernatant/slurry interface du ing natural settling. This quantity was not measured or reported for core samples because the colors of the solids and liquid in the sample were similar, and the interface was not visible during settling tests.

Estimates for the particle settling velocity will be required to complete the design of the scaled experiment. A procedure for estimating the particle settling velocity is provided here. An estimate of the particle size based on core samples will be required to obtain numerical values.

The net gravitational force exerted on a particle immersed in a fluid with a density equal to the density of the slurry mixture is

$$
F=\frac{4}{3}\left(\rho_{s}-\rho_{m}\right) \pi a^{3} g
$$

where $F=$ the force exerted on the particle

$$
\begin{aligned}
\mathrm{a} & =\text { the particle radius } \\
\rho_{\mathrm{m}} & =\text { the density of the slurry mixture } \\
\rho_{\mathrm{s}} & =\text { the density of the solid particle } \\
\mathrm{g} & =\text { the acceleration due to gravity. }
\end{aligned}
$$

The drag force exerted on a small particle is equal to

$$
\mathrm{F}=6 \pi \mu_{\mathrm{e}} \mathrm{a} \mathrm{V}_{\mathrm{s}}
$$


The terminal settling velocity may be determined by balancing the gravitational force and the drag force. This results in

$$
V_{s}=N_{\rho} \frac{2 a^{2} g}{9 v}
$$

where $v$ is the kinematic viscosity of the mixture in which the particle falls.

\subsubsection{Shear Strength}

The shear strength of samples were measured and reported by Tingey (1992, unpublished report). Shear strength is of interest in the nonconvective regions. The magnitudes of the shear strength at $65^{\circ} \mathrm{C}$ for segments 14,19 , and 22 are reported in Table 4.1. On basis of measurements by Tingey, the shear strength of settled samples appears to be approximately equal to $3500 \mathrm{~Pa}$ at $65^{\circ} \mathrm{C}$.

\subsection{ACHIEVING SIMILARITY IN SCALED EXPERIMENTS}

Similarity will be achieved in the model tank and the full scale tank if 1) the two tanks and their jet mitigation equipment are geometrically similar and 2) all dimensionless parameters appearing in the differential equations match during testing. In principle, the criteria may be met at any scale; in practice this is not possible because it is almost certainly impossible to obtain slurries and sludges with effective viscosities less than that of pure water. The properties required at any particular scale will be discussed in Section 4.1.1. The minimum scale at which all parameters may be matched will be discussed in Section 4.2.1.

\subsubsection{Properties Required at an Arbitrary Scale}

The property values required to achieve similarity in a scaled model may be determined by algebraically manipulating the dimensionless parameters appearing in the dimensionless differential equations derived in Section 3.6. The algebraic manipulations performed here assume that the goal is complete similarity. It is possible under special circumstances to make some compromises and to neglect some similarity parameters. This should only be done if there is some analytical or experimental evidence that the effect of a particular similarity parameter is minor. If a decision is made to eliminate an individual similarity parameter, new algebraic manipulation will be required to determine the required parimeters. 
It is useful to define the experimental scale before discussing the property requirements. Let the scale be defined as the ratio of the length scale of the scale model tank to the length scale of SY-101. That is, .

$$
\mathrm{X}=\frac{\mathrm{H}_{\mathrm{OX}}}{\mathrm{H}_{\mathrm{OSY}}}
$$

where the subscript " $\mathrm{x}$ " is used to denote experiments performed in the scaled tank and "SY" is used to denote quantities in the SY-101 itself.

Thus, experiments performed in a tank with linear dims snsions $1 / 12^{\text {th }}$ that of tank $S Y-101$ would have $X=1 / 12$, while experiments performed in a tank with linear dimensions $1 / 4^{\text {th }}$ that of $S Y-101$ will have $X=1 / 4$. Specific examples of scale factor for a $1 / 12^{\text {th }}$ scale tank will be used in this discussion to provide a concrete example of the scale factors and to support discussions of the difficulties involved in designing scaled experiments. The scale factors for $1 / 12^{\text {th }}$ and $1 / 4^{\text {th }}$ scale tanks in tabular form are at the end of this section. These sizes were selected because geometric models of a double-shell tank exist at $1 / 12^{\text {th }}$ scale and a $1 / 4^{\text {th }}$ scale tank is under construction.

The identification of scaled values is in order of increasing complexity and will begin with the four similarity parameters in Table 3.1 that do not contain either the mixture nozzle exit velocity or the length scale. The scaling factor between the magnitude of these properties in the scaled tank and the magnitude in SY-101 is independent of scale. These are

- the mean solids volume fraction $\overline{\varepsilon_{\mathrm{s}}}$

- the functional variation of the effective viscosity $f_{\mu}\left(\tilde{\Delta}, \overline{\varepsilon_{S}}+\Delta \varepsilon_{S}\right)$

- the functional variation settling velocity with concentration $f_{s o}\left(\overline{\varepsilon_{s}}+\Delta \varepsilon_{s}\right)$

- the density ratio $N_{p}=\Delta \rho / \overline{\rho m}$.

The scaling factors of the four quantities defined above are independent of the magnitude of the scale $X$. We will use the subscript $x$ to denote properties of a dynamic simulant used to test the behavior in a scaled test and the subscript SY subscript to denote magnitude in SY-101. Recommendations for matching these quantities are provided below.

The mean solids volume fraction in the scale experiment should match that in SY-101:

$$
\overline{\varepsilon_{S X}}=\overline{\varepsilon_{S} S Y}
$$


Thus, if the mean solids volume fraction in SY-101 is $31 \%$, then a simulant with a solids volume fraction of $31 \%$ should be used during testing.

The form of the viscosity variation with strain rate and solids volume fraction should match

$$
f_{\mu X}\left(\tilde{\Delta}_{\mathbf{X}}, \bar{\varepsilon}_{S}{ }_{X}+\Delta \varepsilon_{S X}\right)=f_{\mu S Y}\left(\tilde{\Delta}_{S Y}, \bar{\varepsilon}_{S} S Y+\Delta \varepsilon_{S S Y}\right)
$$

For example, if the mixture in SY-101 is Newtonian, then the simulant used in testing should be Newtonian. If the mixture is a Bingham plastic, then a Bingham plastic should be used during testing. (See Section 3.3.2 ior an illustration of how to determine the functional form of $f_{\mu}$ for a Bingham plastic.)

The variation of settling velocity with concentration should also match:

$$
f_{S O X}\left(\overline{\varepsilon_{S}} X+\Delta \varepsilon_{S X}\right)=f_{S O} S Y\left(\overline{\varepsilon_{S}} S Y+\Delta \varepsilon_{S S Y}\right)
$$

The variation of settling velocity with concentration for material in SY-101 is unknown. For noncolloidal suspensions, the functional forms must be identical for all mixtures. However, if electrostatic repulsion is important they will not. It is not currently possible to make a direct comparison between settling curve for the simulant and SY-101. Judgment will be required to decide whether the variation of settling velocity with solids volume fraction is acceptable.

The fourth criteria is the match of density parameters $\mathrm{N}_{\rho}$ in the scale model and in SY-101. It is convenient to algebraically manipulate these criteria into a form that is more useful for the purposes of specifying simulant properties.

The mean mixture density is a function of the solids volume fraction, the density of the liquid supernatant, and the density difference between the solids and the liquid:

$$
\overline{\rho_{\mathrm{m}}}=\rho \mathrm{l}+\Delta \rho \overline{\varepsilon_{\mathrm{s}}}
$$

Consequently, the density ratio is

$$
N_{\rho}=\left(\frac{\rho l}{\rho_{S}-\rho l}+\overline{\varepsilon_{S}}\right)^{-1}
$$


If the solids volume density $\overline{\varepsilon_{S}}, x=\overline{\varepsilon_{S}}, S Y$ in the two tanks already matches, then the matching criteria may be expressed as a requirement to match the ratio of the density of the solids to the density of the supernatant or liquid.(a) That is, the ratio

$$
\left(\frac{\rho S}{\rho l}\right)_{X}=\left(\frac{\rho S}{\rho l}\right)_{S Y}
$$

For example, if the magnitude of the density ratio in SY-101 is 1.34 , then the ratio for a simulant used in the scaled experiments should also be 1.34 .

Froude numbers will match provided that the velocity in the scaled experiment is set equal to

$$
W_{0 x}=W_{0, S Y} \sqrt{X}
$$

Froude number equal to those exhibited in the full scale tank may be achieved in a $1 / 12^{\text {th }}$ scale tank by reducing the velocity by a factor of $\sqrt{12}$. Conversely, the scale-up relation to determine the equivalent velocity at full scale is

$$
W_{O, S Y}=W_{o x} / \sqrt{X}
$$

Thus, for example, if mixer pumps can achieve a maximum velocity of $17.9 \mathrm{~m} / \mathrm{s}$, then the effectiveness of pumps running at maximum velocity would be evaluated in the scaled test using a velocity of $17.9 \mathrm{~m} / \mathrm{s} / \sqrt{12}=5.2 \mathrm{~m} / \mathrm{s}$. This would match the correct Froude number.

Because all velocities scale by the same scale factor, ratio of the reference settling velocity must also obey

$$
\mathrm{V}_{\mathrm{SO}, \mathrm{x}}=\mathrm{V}_{\mathrm{soSY}} \sqrt{\mathrm{X}}
$$

(a) The requirement that the solids volume fraction and the density ratio match may also be shown to be equivalent to requiring that the solids concentration, $\bar{C}=\frac{\text { mass solids in tank }}{\text { total mass }}$ and the density ratio $\mathrm{Np}$ also match. Expressing the requirement in this manner simplifies the task of manufacturing the simulant and in no way compromises similarity. 
The correct settling velocity of simulant particles can be determined using this relation, provided that the settling velocity of particles in SY-101 is known. This question must be resolved at a later date.

The inverse time parameter may be used to determine the appropriate scale factor for the period oscillation of a jet or for the period imposed by liquid piston operation:

$$
\eta_{\mathrm{t}}=\frac{\mathrm{H}_{0}}{\mathrm{~T}_{\mathrm{O}} \mathrm{W}_{\mathrm{O}}}
$$

Assuming that the Froude number is matched, the scaling relation for the correct period of operation becomes

$$
T_{o x}=T_{O S Y}\left\{\frac{H_{O X}}{H_{O S Y}} \frac{W_{O S Y}}{W_{O X}}\right\}=T_{O S Y} X^{1 / 2}
$$

For example, if the period for one intake and exhaust stroke of a liquid piston is proposed to be 1 minute, then testing should be performed using a period of 0.29 minutes.

The stress ratio can be matched by selecting an appropriate shear strength. The stress ratio was defined

$$
\mathrm{N}_{\tau}=\frac{\overline{\mathrm{Pm}_{\mathrm{m}}} \mathrm{W}_{\mathrm{o}}^{2}}{\tau_{\mathrm{SS}}}
$$

Assuming that Froude number scaling is being followed, the scale factor for the yield stress of the settled slurry in the model tank is

$$
\left.\left\{\frac{\tau_{S S}}{\overline{\rho m}}\right\}_{X}=\left\{\frac{\tau_{S S}}{\overline{\rho m}}\right\}_{S Y}\left\{\frac{W_{O X}^{2}}{W_{O S Y^{2}}}\right\}=\frac{\tau_{S S}}{\overline{\rho m}}\right\}_{S Y} X
$$


For example, if the shear strength of the settled sludge is 4000 dynes $/ \mathrm{cm}^{2}$ and the average density of the tank contents is $1.6 \times 10^{3} \mathrm{~kg} / \mathrm{m}^{3}$, which results in a ratio of approximately

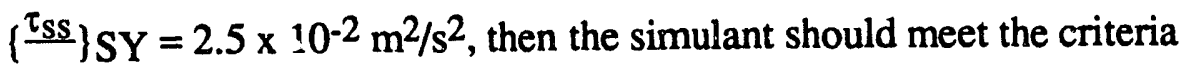
$\overline{\rho \mathrm{m}}$

$$
\left\{\frac{\tau_{\mathrm{ss}}}{\overline{\rho_{\mathrm{m}}}}\right\}_{\mathrm{X}}=2.5 \times 10^{-2} \mathrm{~m}^{2} / \mathrm{s}^{2} \text {. }
$$

The final matching criterion is the Reynolds number. Because the Froude number already matches, it is possible to match both the Reynolds and Froude number by matching the quantity $\mathrm{Re}^{2} / \mathrm{Fr}^{2}$ which is equal to

$$
\mathrm{Re}^{2} / \mathrm{Fr}^{2}=\frac{|\mathrm{g}| \mathrm{H}_{\mathrm{t}}^{3}}{\mathrm{Ve}^{2}}
$$

where $v_{\mathrm{e}}=\mu_{\mathrm{ed}} / \overline{\mathrm{\rho m}_{\mathrm{m}}}$ is the effective kinematic viscosity of the slurry mixture at a reference concentration and strain rate.

Experiments must be performed at the acceleration due to gravity on earth. Consequently, this scale parameter may only be achieved by adjusting the kinematic viscosity as follows:

$$
v_{\mathrm{ex}}=\mathrm{V}_{\mathrm{eSY}} \mathrm{X}^{3 / 2}
$$

Thus, if the SY-101 waste viscosity is approximately $10 \mathrm{cP}$ and its density is $1.5 \times 10^{3} \mathrm{~kg} / \mathrm{m}^{3}$, then assuming that the density of the simulant is also $1.5 \times 10^{3} \mathrm{~kg} / \mathrm{m}^{3}$, the viscosity in the $1 / 12^{\text {th }}$ scale simulant should be $10 \mathrm{cP} / 12^{3 / 2}=0.24 \mathrm{cP}$.

The algebraic relationships in this section can be used to determine the properties required to achieve similarity at any scale. If these properties are used, then any dimensionless parameter may be used to obtain a scale-up criterion. In particular, if the minimum nozzle velocity required to achieve resuspension in the scale model is determined, then minimum velocity required in SY-101 can be calculated using

$$
\mathrm{W}_{\text {omin }}, \mathrm{SY}=\mathrm{W}_{\text {omin },} \mathrm{x} / \sqrt{\mathrm{X}}
$$


However, if the simulant does not have the appropriate properties required by the algebraic relations provided here, then it will not be possible to determine the scale-up ratio on the basis of a similarity analysis alone.

\subsubsection{Strategy for Small Scale Testing}

In principle, similarity may be achieved at any scale if slurries with suitable properties can be manufactured. In practice, exact similarity is not possible when testing is done in a $1 / 12^{\text {th }}$ scale experiment. The impediment to achieving similarity and a strategy for performing experiments at $1 / 12^{\text {th }}$ scale are briefly described here.

In practice, some arbitrary properties are difficult to achieve. A primary impediment to performing tests in an arbitrarily sized tank is producing a simulant with the appropriate viscosity. Other similarity criteria, such as the need to match the correct settling velocity, may also be difficult to achieve but these difficulties are not affected by the experimental scale.

A sample from the convective slurry region of the tank (segment 8) was analyzed to determine its viscosity, solids loading, and particle size.(a) The analysis shows the viscosity of the convective slurry at $65^{\circ} \mathrm{C}$ is $8 \mathrm{cP}$. The bulk density of the convective sample was $1.54 \mathrm{~kg} / \mathrm{m}^{3}$; the corresponding reference kinematic viscosity is $5.2 \times 10^{-6} \mathrm{~m}^{2} / \mathrm{s}$. Similar behavior may be achieved in a $1 / 12^{\text {th }}$ scale tank using a simulant with a kinematic viscosity of $0.12 \mathrm{~m}^{2} / \mathrm{s}$. Assuming that the density of the simulant is also $1.54 \mathrm{~kg} / \mathrm{m}^{3}$, then the dynamic viscosity of the simulant must be $0.2 \mathrm{cP}$.

The viscosity required for $1 / 12^{\text {th }}$ scale testing is relatively low when compared to the viscosity of common liquids. The viscosity of water is approximately $1 \mathrm{cP}$ at $20^{\circ} \mathrm{C}$ and may be reduced somewhat by heating, but it remains above $0.2 \mathrm{cP}$, even at its boiling point of $100^{\circ} \mathrm{C}$. The difficulty of achieving a viscosity of $0.2 \mathrm{cP}$ is even more challenging when considering the solids loading of the sample from segment 8 . Generally, the viscosity of a slurry sample is expected to be greater than that of the pure supernatant. The volume of centrifuged solids at $65^{\circ} \mathrm{C}$ was $20 \%$, while the weight of centrifuged solids was $24 \%$. Consequently, the $0.2 \mathrm{cP}$ criterion must be applied to a sample with a significant volume of solids. It is clear that similarity will be difficult to achieve in very small scale tanks.

Achieving an appropriate slurry viscosity would be simplified by using a mixture with a reference viscosity that is larger than the estimate based on segment 8 data. This would also be useful if suspension of higher viscosity slurries were known to be more difficult than suspension of low viscosity slurries. Unfortunately, although high viscosity leads to a low Reynolds number, which suggests larger regions of laminar flow and lower particle diffusivities, it also leads to lower

(a) Tingey (1992, unpublished report). 
particle settling velocities for a given size particle, which would result in less settling. Consequently, using excessively viscous simulants is no more likely to result in more settling than less settling.

The minimum achievable slurry viscosity at the mean solids concentration of SY-101 is not known. However, it is reasonable to assume that a slurry viscosity of $2 \mathrm{cP}$ may be achieved at elevated temperature. It will be difficult to manufacture slurry with less than $2 \mathrm{cP}$. Estimates of the required slurry viscosities suggest that it will be difficult to achieve complete similarity even at $1 / 4^{\text {th }}$ scale if the viscosity of the mixture in SY-101 is as low as $8 \mathrm{cP}$.

Neither case may be simulated completely in a $1 / 12^{\text {th }}$ scale tank. If experiments are performed in a $1 / 12^{\text {th }}$ scale tank using a viscosity of $2 \mathrm{cP}$, the Reynolds numbers achieved at a particular magnitude of Froude number will be $10 \%$ of the magnitude anticipated in the full scale tank. A synopsis of the scale factor for the properties and variables in a scaled tank is provided in Table 4.2. Because the viscosity is the most difficult variable to scale, the magnitude of sample viscosities in SY-101 are also provided.

A reasonable strategy to overcome the difficulties in achieving complete similarity in scale tanks includes the following:

- Design simulants that match all required variables except the viscosity.

- Select at least two viscosity values to test at a particular scale; these viscosities should be as low as reasonable achievable. This will result in two simulants with different $\mathrm{Re}^{2} / \mathrm{Fr}^{2}=$ $\frac{|\mathrm{g}| \mathrm{H}_{\mathrm{l}}^{3}}{\mathrm{Ve}^{2}}$. The tank will represent a material with a third higher value of $\mathrm{Re}^{2} / \mathrm{Fr}^{2}$.

- Experimentally determine the minimum dimensionless nozzle velocity $W_{0, \text { min }}$ required to achieve off-bottom suspension of the solids in the tank for each simulant.

- Calculate the value of $\mathrm{Fr}_{\min }\left(\frac{|\mathrm{g}| \mathrm{H}_{\mathrm{t}}^{3}}{\mathrm{~V}_{\mathrm{e}}{ }^{2}}\right)$ for each simulant tested in the $\mathrm{X}$ scale tank using

$$
\mathrm{Fr}_{\min }=\mathrm{W}_{0, \min ^{2}} / \mathrm{gl} \mathrm{H}_{\mathrm{t}, \mathrm{x}}
$$

- Perform an extrapolation to determine $\mathrm{Fr}_{\mathrm{min}}, \mathrm{SY}$ for the value of $\operatorname{Re}^{2} / \mathrm{Fr}^{2}$ which represents the tank waste. If two simulants are used at a single scale, linear extrapolation would be performed using the relation

$$
F r_{\operatorname{minSY}}=\mathrm{Fr}_{\min 1}+\frac{\mathrm{Fr}_{\min 1}-\mathrm{Fr}_{\min 2}}{\left(\mathrm{Re}^{2} / \mathrm{Fr}^{2}\right)_{1}-\left(\mathrm{Re}^{2} / \mathrm{Fr}^{2}\right)_{2}}\left\{\left(\mathrm{Re}^{2} / \mathrm{Fr}^{2}\right) S Y-\left(\mathrm{Re}^{2} / \mathrm{Fr}^{2}\right)_{1}\right\}
$$


TABLE 4.2. Properties, Velocity, and Length Scale Factors Required to Achieve Similarity at $1 / 12^{\text {th }}$ and $1 / 4^{\text {th }}$ Scale. (Conversions of dynamic viscosities assume that the mixture density matches in both tanks.)

\begin{tabular}{|c|c|c|c|c|}
\hline 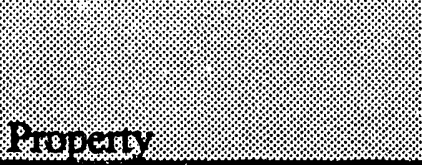 & $101-5 v^{2}$ & 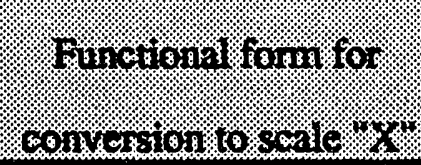 & $x=112$ & $x=1 / 4$ \\
\hline Length scale & $\mathrm{H}_{\mathrm{t}} \mathrm{SY}$ & $\mathrm{H}_{\mathrm{t} S \mathrm{Y}} \mathrm{X}$ & $\frac{\mathrm{H}_{\mathrm{tSY}}}{12}$ & $\frac{\mathrm{H}_{\mathrm{tSY}}}{4}$ \\
\hline $\begin{array}{l}\text { Ratio of shear strength } \\
\text { to mixture density } \\
\left\{\tau_{\mathrm{ss}} / \overline{\rho_{\mathrm{m}}}\right\}\end{array}$ & $\left\{\tau_{\mathrm{SS}} / \overline{\rho_{\mathrm{m}}}\right\} \mathrm{SY}$ & $\left\{\tau_{\mathrm{SS}} / \overline{\rho_{\mathrm{m}}}\right\} \mathrm{SY} X$ & $\frac{\left\{\tau_{\mathrm{ss}} / \overline{\rho_{\mathrm{m}}}\right\}_{\mathrm{SY}}}{12}$ & $\frac{\left\{\tau_{\mathrm{ss}} / \overline{\rho_{\mathrm{m}}}\right\}_{\mathrm{SY}}}{4}$ \\
\hline Kinematic viscosity & vSY & vSY $X^{3 / 2}$ & $\frac{V S Y}{42 .}$ & $\frac{v S Y}{8}$ \\
\hline Particle settling velocity & $V_{\text {SOSY }}$ & $\mathrm{V}_{\text {SoSY }} \mathrm{X}^{1 / 2}$ & $\frac{\mathrm{V}_{\text {SOSY }}}{3.5}$ & $\frac{\mathrm{V}_{\mathrm{SOSY}}}{2}$ \\
\hline Period of oscillation & $\mathrm{T}_{0} \mathrm{SY}$ & $\mathrm{T}_{\mathrm{OSY}} \mathrm{X}^{1 / 2}$ & $\frac{T_{0 S Y}}{3.5}$ & $\frac{T_{\mathrm{OSY}}}{2}$ \\
\hline Nozzle velocity & $\mathrm{W}_{\mathrm{OSY}}$ & $\mathrm{W}_{\mathrm{om}} \mathrm{X}^{1 / 2}$ & $\frac{W_{0}}{3.5}$ & $\frac{W_{0}}{2}$ \\
\hline Solids volume fraction & $\overline{\varepsilon_{s}}$ & $\overline{\varepsilon_{s}}$ & $\overline{\varepsilon_{\mathbf{s}}}$ & $\overline{\varepsilon_{S}}$ \\
\hline Density ratio $\mathrm{N}_{\rho}$ & $\mathrm{N} \rho S \mathrm{Y}$ & $\mathrm{N}_{\rho S Y}$ & $\mathrm{~N}_{\rho S Y}$ & $\mathrm{~N}_{\rho S Y}$ \\
\hline 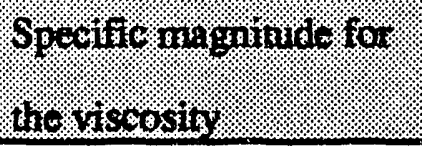 & & & & \\
\hline $\begin{array}{l}\text { Kinematic viscosity, } \\
\text { veSY }\end{array}$ & $5.2 \mathrm{~m}^{2} / \mathrm{s}$ & vesY $X^{3 / 2}$ & 0.125 & 0.65 \\
\hline Dynamic viscosity $\mu_{\mathrm{m}}$ & $8 \mathrm{cP}$ & & $0.2 \mathrm{cP}$ & $1.25 \mathrm{cP}$ \\
\hline Dynamic viscosity $\mu_{\mathrm{m}}$ & $40 \mathrm{cP}$ & & $0.96 \mathrm{cP}$ & $5 \mathrm{cP}$ \\
\hline
\end{tabular}


Here the subscripts 1 and 2 refer to the first simulant tested and the second simulant tested, respectively.

- Calculate the predicted nozzle velocity for SY-101 using the relation

$$
W_{O, \min } S Y=\sqrt{F_{r_{\min S Y}}|g| H_{S Y}}
$$

This strategy may be used to evaluate all proposed hydraulic mitigation strategies. Identical simulants and model tanks should be used for all tests performed at identical scale $X$. Identical simulants are required to ensure that the same magnitudes of the similarity parameters are achieved. Identical model tanks will ensure that no differences in tank geometry or scale affect the results.

It should be emphasized that interpolation of data is generally more accurate than extrapolation. However, interpolation is not possible in this case. The dangers of extrapolation may be minimized by limiting the range of extrapolation, or by using more than two simulant properties, because this will allow nonlinear extrapolation to be performed. 


\subsection{REEERENCES}

Allemann, R. T., Z. I. Antoniak, L. L. Eyler, and L. M. Liljegren. 1991. Conceptual Models for Waste Tank Mechanistic Analysis Status Report - January 1991. PNL-8011, Pacific Northwest Laboratory, Richland, Washington.

Babad, H., J. L. Deichman, B. M. Johnson, D. K. Lemon, and D. M. Strachan. 1991. Mitigation/ Remediation Concepts for Hanford Hydrogen Gas Generating Waste Tanks. WHCEP-0516, Westinghouse Hanford Company, Richland, Washington.

Bamberger, J. A., P. S. Lowery, and L. M. Liljegren. 1990. A Methodology to Predict the Uniformity of Double-Shell Tank Waste Slurries Based on Mixing Pump Operation. PNL-7665. Pacific Northwest Laboratory, Richland, Washington.

Fossett, H., and L. E. Prosser. 1949. "The Application of Free Jets to the Mixing of Fluids in Bulk". Proc. I. Mech. E. 160:224-254.

Fossett, H., and L. E. Prosser. 1951. "The Application for Free Jets to the Mixing of Fluids in Bulk." Tran. Inst. Chem. Engr. 29:322.

Fox, R. W., and A. T. McDonald. 1978. Introduction to Fluid Mechanics. 2nd Edition, John Wiley and Sons, New York.

Leinhard, J. H. 1981. A Heat Transfer Textbook. Prentice Hall, Englewood Cliffs, New Jersey.

Sha, W. T., and S. L. Soo. 1979. "On the Effect of the $\triangle \mathrm{P}$ Term in Multiphase Mechanics." Int. J. Multiphase Flow 5:153-158.

White, F. M. 1979. Fluid Mechanics. 2nd Edition. McGraw Hill, New York.

Yih, C. S. 1979. Fluid Mechanics. West River Press, Ann Arbor, Michigan. 


\section{APPENDIX A}

EXAMPLE OF THE DIEEICULTIES ASSOCIATED WITH SCALE-UP OE DATA FROM NONSIMILAR EXPERIMENTS 


\section{APPENDIX A \\ EXAMPLE OF THE DIFEICULTIES ASSOCIATED WITH SCALE-UP OE DATA FROM NONSMMUAREXPERIMENTS}

By using a well documented example, this appendix illustrates the inherent difficulties associated with scale-up of data from nonsimilar experiments. The example was selected because it is characterized by two independent dimensionless parameters rather than thirteen.

Suppose that an experiment is performed to determine the convective heat transfer coefficient $\mathrm{h}$ when fluid flows at velocity $\mathrm{U}$ past a cylinder with diameter $\mathrm{D}$. Scaling law analysis indicates that the dimensionless heat transfer coefficient or Nusselt number, NuD, can be determined on the basis of two parameters $\mathrm{NuD}_{\mathrm{D}}=f(\mathrm{Pr}, \mathrm{Re})$ where $f$ is some unknown functional form. Here the Nusselt number, Prandtl number, and Reynolds number are defined $\mathrm{Nu}_{D}=h D / k, \operatorname{Pr}=\alpha / v$, and $\operatorname{Re}$ $=U D / N$, respectively. The properties of the fluid flowing past the plate are thermal conductivity $\mathrm{k}$, kinematic viscosity $v$, thermal diffusivity $\alpha$, and density $\rho$.

Experiments are required to determine the functional form of the relationship. If heat transfer coefficients are desired for exactly one case, one experiment could be performed at the appropriate Prandtl and Reynolds, and then the appropriate value of NuD could be determined from experimental measurement. The scale-up relationship of a test performed at small scale which matches the Reynolds and Prandtl of the full scale application would be

$$
\mathrm{Nu}_{\text {full scale }}=\mathrm{Nu}_{\text {test scale }}
$$

This relation would hold if and only if the similarity criteria were met. That is, if tests were performed at $\operatorname{Pr}_{\text {full scale }}=\operatorname{Pr}_{\text {test scale }}$ and $\operatorname{Re}_{\text {full scale }}=\mathrm{Re}_{\text {test scale. }}$

The scale-up relation could also be written as:

$$
\frac{h_{\text {full scale }}}{h_{\text {test scale }}}=\frac{k_{\text {full scale }} D_{\text {test scale }}}{k_{\text {test scale }} D_{\text {full scale }}}
$$

Thus, dimensional analysis provides both the criterion for performing scaled experiments (i.e., both $\mathrm{Re}$ and $\mathrm{Pr}$ are matched) and the scale-up relationship between experiments (i.e., that $\mathrm{Nu}$ will match). 
This particular example was selected because experimental data establishing the form of the function $f$ already exist. A broad study by Churchill and Bernstein (1977) shows that data can be fit using

$$
\left.N u_{D}=0.3+\frac{0.62 \operatorname{ReD}^{0.5 P_{r}^{0}} 0.333}{\left(1+(0.4 / \operatorname{Pr})^{0.667}\right\}^{0.25}}\left(1+\frac{\operatorname{Rep}_{D}}{282000}\right) \cdot 525\right\}^{0.8}
$$

Performing a single experiment at the correct Prandel number and Reynolds number is the experimental equivalent of evaluating the experimentally determined general expression, and it results in the correct dimensionless Nusselt number. However, if only one parameter (Reynolds or Prandtl) is matched experimentally (or worse still, none), then the incorrect $\mathrm{Nu}$ will be obtained, and virtually nothing could be said about the relationship between the measured convective heat transfer coefficient in a scaled experiment and a full scale application. This is why data collected using water alone cannot be usefully applied to predict heat transfer in sodium loops-the Prandtl number of sodium differs markedly from that of water.

There is no conceptual difference between simply inserting the incorrect magnitudes of Re and $\mathrm{Pr}$ to calculate $\mathrm{Nu}$ on the basis of the functional relationship and performing the experiment at incorrect values of the dimensionless parameters. There is, however, a significant difference in cost.

\section{REFERENCE}

Churchill, S.W., and M. Bernstein. 1977. "A Correlation Equation for Forced Convection from Gases and Liquids to a Circular Cylinder in Crossflow." J. Heat Transfer. Trans, ASME, Ser. C, 99:300-306. 


\section{APPENDIX B}

PARTICLEDIEEUSIVITY 


\section{APPENDIX B}

\section{PARTICLEDIFEUSIVITY}

Particle diffusivity, which is a complex function of the flow field, is important in mathematical descriptions of the behavior of slurries. Factors which affect the particle diffusivity are discussed extensively in the literature. Notable analyses include those by Lumley (1957), Chao (1964), Tchen (1947), Reeks (1977), and Pismen and Nir (1978 and 1979).

For the purposes of the analysis here, it is sufficient to state that 1) both particle and fluid diffusivities are dependent parameters in any large-scale flow, 2) the fluid diffusivity scales as the product of the characteristic velocity and the integral length scale of the fluid turbulence (both are dependent parameters during any hydraulic mitigation application), and 3) the particle diffusivity is proportional to the fluid diffusivity, but it is also affected by factors that govern the response of small particles to turbulent motions.

When small particles are suspended in single phase flow, the relative motion of the particles to the fluid is a function of the particle response time, the time scale of the turbulent fluctuations, and the densities of the particles and the fluid through which it moves. Thus, the local particle diffusivity may be written in the form

$$
\mathcal{D}_{\mathrm{p}}=\mathrm{w}^{\prime} \mathbf{l} \mathbf{f}_{\mathcal{D} 1}\left(\tau_{\mathrm{p}} / \tau_{\mathrm{f}}, \Delta \rho / \mathrm{Pm}_{\mathrm{m}}\right)
$$

where

$$
\begin{aligned}
w^{\prime} & =\text { the characteristic velocity of the turbulence fluctuations } \\
1 & =\text { the characteristic length scale of the turbulence fluctuations } \\
\tau_{\mathrm{f}} & =\text { the characteristic time scale of the fluid turbulence }
\end{aligned}
$$

$\mathrm{f}_{\mathcal{D}}\left(\tau_{\mathrm{p}} / \tau_{\mathrm{f}}, \Delta \mathrm{p} / \mathrm{p}_{\mathrm{m}}\right)=$ a dimensionless function which describes the relationship between the fluid turbulence structure and the particle diffusivity.

In terms of a large-scale application, the only independent parameters are the particle response time $\tau_{\mathrm{p}}$ and the density ratio $\Delta \mathrm{p} / \mathrm{pm}_{\mathrm{m}}$. All other parameters arise as a result of flow in the mixture, and consequently, the function $\mathrm{f}_{\mathcal{D}}$ will scale appropriately if all identifiable conditions for similarity are achieved. 
Generally, the magnitude of both the turbulent velocity fluctuations and the length scales are functions of the Reynolds number, and it is possible to rewrite the diffusivity relation in dimensionless form as

$$
\frac{\mathcal{D}_{\mathrm{p}}}{\mathrm{W}_{\mathrm{O}} \mathrm{H}_{\mathrm{t}}}=\mathrm{f}_{\mathcal{D}}\left(\tau_{\mathrm{p}} / \mathrm{t}_{\mathrm{f}}, \Delta \rho / \rho_{\mathrm{m}}, \operatorname{Re}_{\mathrm{H}}\right)
$$

where $W_{0}$ is a characteristic velocity for the overall flow field and $\mathrm{H}_{t}$ is a characteristic length scale for the overall flow. In this particular case, the chosen characteristic velocity is the jet nozzle velocity, and the chosen characteristic length scale is the tank height. The particular form of the function $\mathrm{f}_{\mathcal{D}}$ will be affected by geometric features as well.

The relevance of the final functional form to the design of hydraulic mitigation experiments is that the correct particle diffusivity can be obtained if the appropriate Reynolds number, density ratio, and ratio of particle response time to turbulence time scale can be maintained. This can be ensured by matching the similarity parameters identified in Section 3.7.

\section{REFERENCES}

Chao, B. T. 1964. "Turbulent Transport Behavior of Small Particles in Dilute Suspension." Osterreichisces Ingenieur-Archiv 18:7-21.

Lumley, J. L. 1957. Some Problems Connected with the Motion of Small Particles in Turbulent Fluid. Ph.D. Dissertation, Johns Hopkins University, Baltimore, Maryland.

Nir, A., and L. M. Pismen. 1979. "The Effect of a Steady Drift on the Dispersion of a Particle in a Turbulent Fluid." J. Fluid Mech 94:369-381.

Pismen, L. M., and A. Nir. 1978. "On the Motion of Suspended Particles in Stationary Homogenous Turbulence." J. Fluid Mech 84:193-221.

Reeks, M. W. 1977. "On the Dispersion of Small Particles Suspended in an Isomorphic Turbulent Fluid." J. Fluid Mech 83:529-546.

Tchen, C. M. 1947. "Mean Value and Correlation Problems Connected with the Motion of Small Particles Suspended in a Turbulent Fluid." Chapter 4 in Marinus Nijhoff, The Hague. West River Press. 
APPENDIX C

PROBLEMS ASSOCIATED WITH CODE VALIDATION USING EXPERIMENTS WITHNODENSITY STRATIEICATION 


\section{APPENDIX C \\ PROBLEMS ASSOCLATED WITH CODE VALIDATION USING EXPERIMENTS WITH NO DENSITY STRATIFICATION}

Errors inherent in extrapolation have an analog in numerical ana'vsis. The case of turbulence is an example in which code extrapolation is problematic. A eode may successfully predict phenomena in a laminar flow but fail completely for a turbulent flow. This is because codes require a submodel to predict Reynolds stresses or velocity cross-correlations that exist in turbulent flow. Because the Reynolds stresses are identically zero in laminar flows, this submodel is not required for laminar flow. Consequently, inadequacies in the Reynolds stress submodel cannot be detected by comparing numerical predictions to laminar flow data.

In the problem addressed here, difficulties can arise when data collected in unstratified flows is used to validate numerical results which will be applied in stratified flows. Crosscorrelations between density fluctuations appear in the equations describing transport of turbulent kinetic energy. Additional closure models are required to estimate the magnitude of these crosscorrelations. Because the density-velocity cross-correlation is equal to zero in the absence of density gradients, these closure models cannot be validated on the basis of data collected in unstratified flows.

C. 1 


\section{DISTRIBUTION}

No. of

Copies

\section{OFESITE}

12 DOE/Office of Scientific and Technical Information

C. S. Abrams

1987 Virginia

Idaho Falls, ID 83404

E. C. Ashby

225 North Avenue

Boggs Chemistry Building

Georgia Institute of Technology

Atlanta, GA 30332

N. E. Bibler

Westinghouse Savannah River Co.

Bldg. 773A, Room B132

Box 616

Aiken, SC 29802

D. Campbell

102 Windham Road

Oak Ridge, TN 37830

F. N. Carlson

6965 North, 5th West

Idaho Falls, ID 83401

G. R. Choppin

Department of Chemistry B-164

The Florida State University

Tallahassee, FL 32306

M. First

Harvard School of Public Health

665 Huntington Avenue

Boston, MA 02115

C. W. Forsberg

Oak Ridge National Laboratory

MS-6495, P.O. Box 2008

Oak Ridge, TN 37831-6495
No. of

Copies

C. Grelecki

Hazards Research Corporation

200 Valley Road, Suite 301

Mt. Arlington, NJ 07856

E. P. Horwitz

Chemistry Division

Argonne National Laboratory

9700 Cass Avenue

Argonne, IL 60439-4831

B. C. Hudson

Lawrence Livermore National Laboratory

Nuclear Test Containment Program

P.O. Box 808, L-221

East Avenue

Livermore, CA 94550

B. R. Kowalski

Chemistry Department BG-10

University of Washington

Seattle, WA 98195

6 Los Alamos National Laboratory

P.O. Box 1663

Los Alamos, NM 87545

Attn: L. H. Sullivan, K557

J. R. White, K555

S. W. Eisenhawer, K557

J. Edwards, K557

J. Hanson, H5-09

T. E. Larson, P915

J. L. Mai

723 45th Avenue

San Francisco, CA 94121

D. Meisel

Chemistry Department

Argonne National Laboratory

9700 Cass Avenue

Argonne, IL 60439-4831 
No. of

Copies

F. L. Parker

Vanderbilt University

P.O. Box 1596, Station B

Nashville, TN 37235

G. Powers

Design Science Inc.

163 Witherow Road

Sewickley, PA 15143

G. A. Russell

Professor of Chemistry

Iowa State University

Gilman Hall

Ames, IA 50011-3111

A. Schneider

Massachusetts Institute of Technology

Department of Nuclear Engineering

Room 24-1098

77 Massachusetts Avenue

Cambridge, MA 02139

W. W. Schulz

727 Sweetleaf Drive

Wilmington, DE 19808

D. D. Siemer

WINCO

IRC MS 2207

Idaho Falls, ID 83403

S. E. Slezak

Sandia National Laboratories

1515 Eubank NE

Division 6424

P.O. Box 5800

Albuquerque, NM 87185

W. J. Thomson

Dept. of Chemical Engineering

Washington State University

Pullman, WA 99164
No. of

Copies

J. Tseng

U.S. Department of Energy

EM-35

Trevion II

Washington, D.C. 20585-0002

G. Wallis

Associate Dean

Thayer School of Engineering

Dartmouth College

Hanover, NH 03755

\section{ONSTTE}

4 DOE Richland Field Office

R. F. Christensen, A4-02

R. E. Gerton, A5-21

J. M. Gray, A4-02

G. W. Rosenwald, A5-21

31 Westinghouse Hanford Company

H. Babad, B2-15

T. R. Beaver, H0-33

T. R. Benegas, H5-09

H. R. Brager, L5-03

T. M. Burke, H0-34

R. J. Cash, R2-31

S. C. Chang, H0-34

C. DeFigh-Price, R2-31

J. C. Fulton, R2-31

K. A. Gasper, B3-68

M. N. Hall, H5-68

C. E. Hanson, H5-09

G. D. Johnson, L5-03

N. W. Kirch, R2-11

W. L. Knecht, H0-34

J. W. Lentsch, R2-31

R. D. Marusich, H5-32

G. J. Miskho, R2-50

C. P. Molteni, R1-51

D. Ogden, H0-34

M. A. Payne, R2-50 
No. of

Copies

R. W. Reed, R1-51

D. A. Reynolds, R2-11

D. C. Richardson, R2-31

K. Sathyanarayana, H0-34

M. H. Shannon, B1-35

D. D. Stepnewski, N1-31

T. I. Stokes, H5-09

J. D. Thomson, R1-30

R. E. Vandercook, S6-07

D. D. Wodrich, R2-23

34 Pacific:Northwest Laboratory

R. T. Allernann, K5-19

J. A. Bamberger, K7-15

J. M. Bates, K7-15

J. B. Colson, K5-10

M. R. Elmore, P7-19
No. of

Copies

C. W. Enderlin, K7-15

E. J. Eschbach, K7-15

L. L. Eyler, K7-15

J. A. Fort, K7-15 (5)

M. S. Greenwood, K2-31

M. S. Hanson, K1-51

B. M. Johnson, Jr., K5-02 (3)

M. R. Kreiter, K7-90 (3)

L. M. Liljegren, K7-15

N. J. Lombardo, K7-02

T. E. Michener, K7-15

R. D. Scheele, P7-25

D. M. Strachan, K2-44

D. S. Trent, K1-82

H. H. Van Tuyl, P7-22

Publishing Coordination

Technical Report Files (5) 

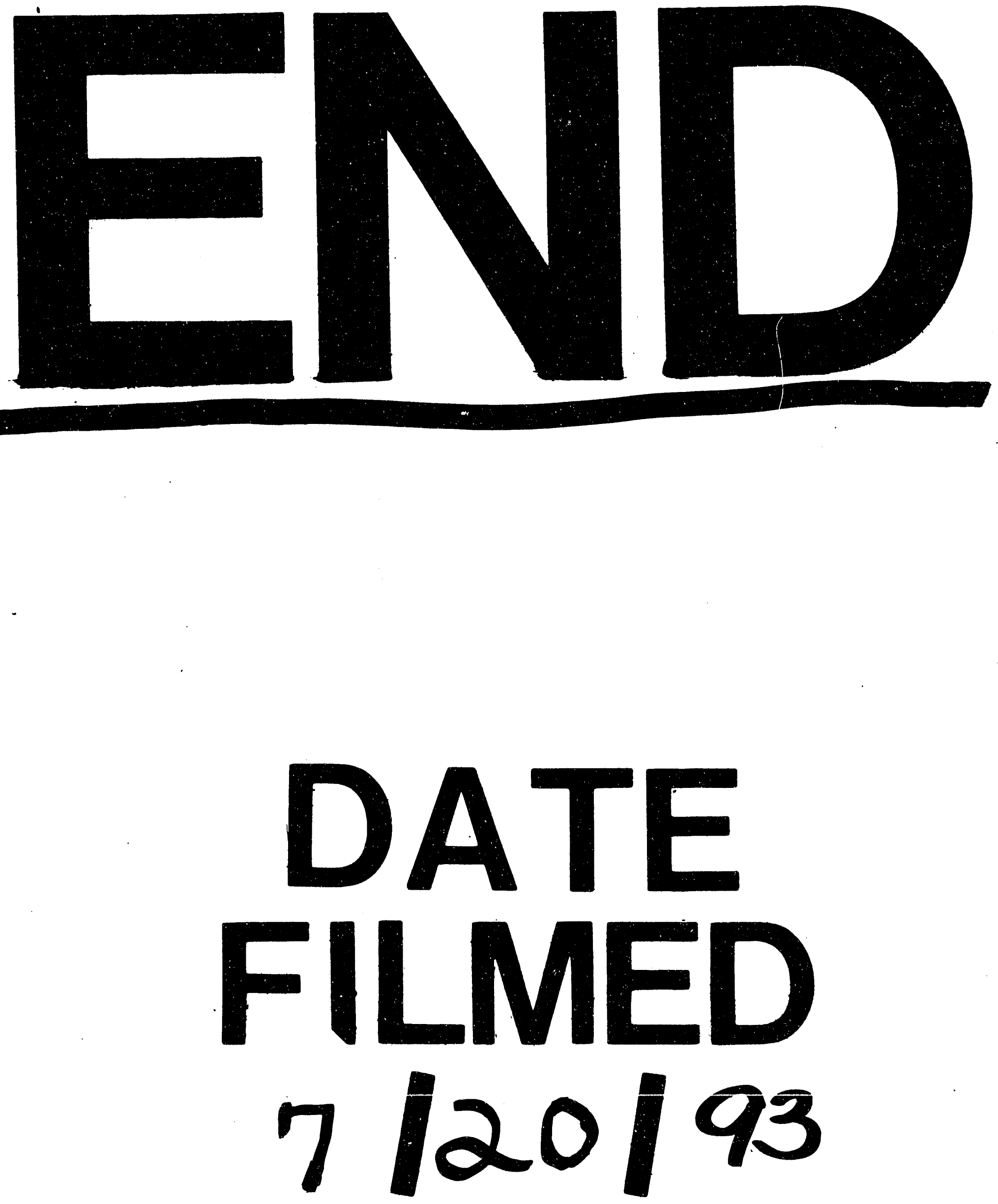
